\title{
Olefination of Ketones Using A Gold(III)-Catalyzed Meyer-Schuster Rearrangement
}

\author{
Douglas A. Engel and Gregory B. Dudley* \\ Department of Chemistry and Biochemistry, Florida State University, \\ Tallahassee, Florida 32306
}

\section{Supporting Information}

\section{CONTENTS:}

General experimental procedures

S1-S4

Characterization (analytical) data

S5-S12

Copies of ${ }^{1} \mathrm{H}$ NMR and ${ }^{13} \mathrm{C}$ NMR spectra

S13-S38

\section{GENERAL EXPERIMENTAL PROCEDURES}

${ }^{1} \mathrm{H}-\mathrm{NMR}$ and ${ }^{13} \mathrm{C}$-NMR spectra were recorded on $300 \mathrm{MHz}$ spectrometer using $\mathrm{CDCl}_{3}$ as the deuterated solvent. The chemical shifts $(\delta)$ are reported in parts per million (ppm) relative to the residual $\mathrm{CHCl}_{3}$ peak (7.26 ppm for ${ }^{1} \mathrm{H} \mathrm{NMR}, 77.0 \mathrm{ppm}$ for ${ }^{13} \mathrm{C} \mathrm{NMR}$ ). The coupling constants $(J)$ were reported in Hertz $(\mathrm{Hz})$. IR spectra were recorded on an FTIR spectrometer on $\mathrm{NaCl}$ discs. Mass spectra were recorded using chemical ionization (CI) or electron ionization (EI) techniques. Yields refer to isolated material judged to be $\geq 95 \%$ pure by ${ }^{1} \mathrm{H}$ NMR spectroscopy following silica gel chromatography. All chemicals were used as received unless otherwise stated. Tetrahydrofuran (THF) was purified by passing through a column of activated alumina. The $n$-BuLi solutions were titrated with menthol dissolved in tetrahydrofuran using 1,10-phenanthroline as the 
indicator. The purifications were perfomed by flash chromatography using silica gel F254 (230-499 mesh particle size).

\section{Typical procedure for the preparation of phenyl propargylic alcohols $2 \mathrm{a}, 2 \mathrm{~b}, 2 \mathrm{c}$}

(Table 1: entries 1-3).

To a THF solution $(10 \mathrm{~mL})$ of phenylacetylene $(1.55 \mathrm{~mL}, 14.1 \mathrm{mmol})$ was added $n$-BuLi (4.6 $\mathrm{mL}, 11 \mathrm{mmol}, 2.3 \mathrm{M})$ dropwise over $5 \mathrm{~min}$ at $-78^{\circ} \mathrm{C}$ under argon atmosphere. The solution was allowed to warm to $0{ }^{\circ} \mathrm{C}$ over $1 \mathrm{~h}$ and held at $0{ }^{\circ} \mathrm{C}$ for an additional $30 \mathrm{~min}$. The solution was then recooled to $-78^{\circ} \mathrm{C}$ and 3 -pentanone $(0.75 \mathrm{~mL}, 7.1 \mathrm{mmol})$ was added in one portion. The solution was allowed to warm to room temperature over $1 \mathrm{~h}$ and held at room temperature for an additional $3 \mathrm{~h}$. Saturated aqueous $\mathrm{NH}_{4} \mathrm{Cl}$ solution was added to quench the reaction and the mixture was extracted with ethyl acetate. The organic layer was washed with water, saturated aqueous sodium bicarbonate, and brine. The organic layer was dried over $\mathrm{MgSO}_{4}$, filtered, and concentrated. The residue was purified using silica gel column chromatography (hexanes/ethyl acetate $=20 / 1-10 / 1)$ to give 3-ethyl-1-phenyl-pent-1-yn-3-ol (2b) in 100\% yield (1.33g).

\section{Typical procedure for the preparation of ethoxy propargylic alcohols $2 \mathrm{~d}, \mathbf{2 e}, \mathbf{2 f}, \mathbf{2 g}$}

\section{(Table 1: entries 4-8, Table 2: entries 1-8).}

To a THF solution $(7 \mathrm{~mL})$ of ethyl ethynyl ether $(0.7 \mathrm{~g}$, ca. $40 \%$ by weight in hexanes, ca. 9 mmol was added $n$-BuLi $(1.5 \mathrm{~mL}, 3.4 \mathrm{mmol}, 2.3 \mathrm{M})$ dropwise over $5 \mathrm{~min}$ at $-78{ }^{\circ} \mathrm{C}$ under argon atmosphere. The solution was allowed to warm to $0{ }^{\circ} \mathrm{C}$ over $1 \mathrm{~h}$ and held at $0{ }^{\circ} \mathrm{C}$ for an additional $30 \mathrm{~min}$. The solution was then recooled to $-78{ }^{\circ} \mathrm{C}$ and pinacolone 
$(0.30 \mathrm{~mL}, 2.4 \mathrm{mmol})$ was added in one portion. The solution was allowed to warm to room temperature over $1 \mathrm{~h}$ and held at room temperature for an additional $3 \mathrm{~h}$. Saturated aqueous $\mathrm{NH}_{4} \mathrm{Cl}$ solution was added to quench the reaction and the mixture was extracted with ethyl acetate. The organic layer was washed with water, saturated aqueous sodium bicarbonate, and brine. The organic layer was dried over $\mathrm{MgSO}_{4}$, filtered, and concentrated. The residue was purified using silica gel column chromatography (hexanes/ethyl acetate $=20 / 1-7 / 1$ ) to give 1-ethoxy-3-methyl-3-tert-butyl-1-propyn-3-ol $(\mathbf{2 g})$ in $83 \%$ yield $(0.34 \mathrm{~g})$.

\section{Typical procedure for preparation of $\alpha, \beta$-unsaturated esters $3 \mathrm{a}-3 \mathrm{~g}$}

(Table 1: entries 1-8, Table 2: entries 1-7)

To a $\mathrm{CH}_{2} \mathrm{Cl}_{2}$ solution (8 $\mathrm{mL}$ ) of 1-ethoxy-3-methyl-3-tert-butyl-1-propyn-3-ol (2g) in an open flask was added $95 \%$ ethanol $(0.16 \mathrm{~mL}, 2.9 \mathrm{mmol})$ followed by $\mathrm{AuCl}_{3}(35 \mathrm{mg}, 0.12$ mmol, fine powder). After $5 \mathrm{~min}$, the reaction mixture was filtered through a plug of $\mathrm{SiO}_{2}$ with the aid of an ethyl acetate/hexane mixture (1/7). The filtrate was concentrated and purified using silica gel column chromatography (hexanes/ethyl acetate $=50 / 1$ ) to give 3,4,4-trimethyl-pent-2-enoic acid ethyl ester $(\mathbf{3 g})$ in $86 \%$ yield (85 $\mathrm{mg}$ ).

\section{Typical two step procedure for preparation of $\boldsymbol{\alpha}, \boldsymbol{\beta}$-unsaturated esters $3 \mathrm{~d}, \mathbf{3 f}-\mathbf{3 j}$}

(Table 3: entries 1-6)

To a THF solution $(2.6 \mathrm{~mL})$ of ethyl ethynyl ether $(0.13 \mathrm{~g}$, ca. $40 \%$ by weight in hexanes, ca. $2 \mathrm{mmol})$ was added $n$-BuLi $(0.40 \mathrm{~mL}, 0.75 \mathrm{mmol}, 2.0 \mathrm{M})$ dropwise over $5 \mathrm{~min}$ at $-78{ }^{\circ} \mathrm{C}$ under argon atmosphere. The solution was allowed to warm to $0{ }^{\circ} \mathrm{C}$ over $1 \mathrm{~h}$ and 
held at $0{ }^{\circ} \mathrm{C}$ for an additional $30 \mathrm{~min}$. The solution was then recooled to $-78^{\circ} \mathrm{C}$ and 2adamantanone ( $75 \mathrm{mg}, 0.5 \mathrm{mmol}$ ) was added in one portion. The solution was allowed to warm to room temperature over $1 \mathrm{~h}$ and held at room temperature for an additional $3 \mathrm{~h}$. Saturated aqueous $\mathrm{NH}_{4} \mathrm{Cl}$ solution was added to quench the reaction and the mixture was extracted with ethyl acetate. The organic layer was washed with water, saturated aqueous sodium bicarbonate, and brine. The organic layer was dried over $\mathrm{MgSO}_{4}$, filtered, and concentrated. To the concentrated mixture in an open flask was added $6 \mathrm{~mL}$ of $\mathrm{CH}_{2} \mathrm{Cl}_{2}$, followed by $95 \%$ ethanol $(0.14 \mathrm{~mL}, 2.5 \mathrm{mmol})$ and $\mathrm{AuCl}_{3}(7.6 \mathrm{mg}, .025 \mathrm{mmol}$, fine powder). [NOTE: $15.2 \mathrm{mg}, 0.05 \mathrm{mmol}$ of $\mathrm{AuCl}_{3}$ was used for the preparation of $\mathbf{3 j}$ and 3k.] After $30 \mathrm{~min}$, the reaction mixture was filtered through a plug of $\mathrm{SiO}_{2}$ with the aid of an ethyl acetate/hexane mixture (1/7). The filtrate was concentrated and purified using silica gel column chromatography (hexanes/ethyl acetate $=50 / 1$ ) to give adamantan-2ylidene-acetic acid ethyl ester (3f) in 99\% yield (109 mg) over two steps. 


\section{Analytical Data}

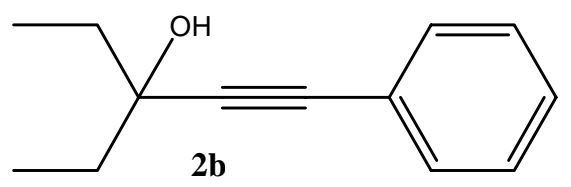

3-ethyl-1-phenyl-pent-1-yn-3-ol (2b): light yellow oil; ${ }^{1} \mathrm{H} \mathrm{NMR}\left(300 \mathrm{MHz}, \mathrm{CDCl}_{3}\right) \delta$ $1.11(\mathrm{t}, J=7.4 \mathrm{~Hz}, 6 \mathrm{H}), 1.77(\mathrm{q} \mathrm{d}, J=7.4,4.5 \mathrm{~Hz}, 4 \mathrm{H}), 1.94($ br s, $1 \mathrm{H}), 7.28-7.33(\mathrm{~m}$, 3H), 7.40-7.44 (m, 2H). For full characterization refer to Galli, C. et al. J. Org. Chem. 1994, 59, 6786-6795.

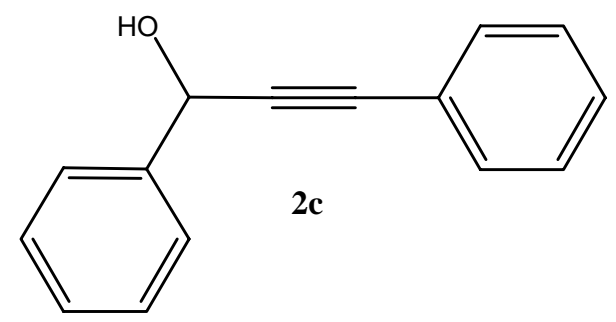

1,3-diphenyl-prop-2-yn-1-ol (2c): colorless oil; NMR $\left(300 \mathrm{MHz}, \mathrm{CDCl}_{3}\right) \delta 2.27(\mathrm{~d}, J=$ 8.1 Hz, 1H), $5.70(\mathrm{~s}, 1 \mathrm{H}), 7.31-7.50(\mathrm{~m}, 8 \mathrm{H}), 7.62-7.65(\mathrm{~m}, 2 \mathrm{H}) ;{ }^{13} \mathrm{C} \mathrm{NMR}(75 \mathrm{MHz}$ $\left.\mathrm{CDCl}_{3}\right) \delta 64.6,86.3,88.9,122.3,126.6,128.1,128.3,128.4,131.5,140.5 ;$ IR (neat) $3350,3062,2874,2229,1953,1884,1807,1598,1490,1384,1280,1190,1030,917$, 756, $691 \mathrm{~cm}^{-1}$; HRMS (CI) Calcd for $\mathrm{C}_{15} \mathrm{H}_{12} \mathrm{O}\left(\mathrm{M}-\mathrm{H}^{+}\right)$207.0810. Found 207.0808. 


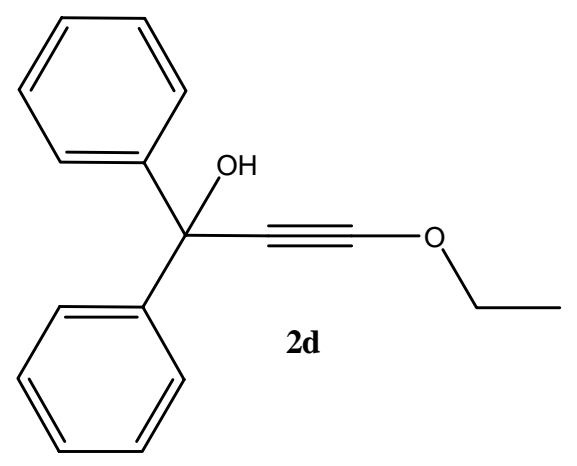

3-ethoxy-1,1-diphenyl-prop-2-yn-1-ol (2d): yellow oil; ${ }^{1} \mathrm{H}$ NMR $\left(300 \mathrm{MHz}, \mathrm{CDCl}_{3}\right) \delta$ $1.41(\mathrm{t}, J=7.1 \mathrm{~Hz}, 3 \mathrm{H}), 2.64(\mathrm{~s}, 1 \mathrm{H}), 4.18(\mathrm{q}, J=7.1 \mathrm{~Hz}, 2 \mathrm{H}), 7.21-7.34(\mathrm{~m}, 6 \mathrm{H}), 7.60-$ 7.63 (m, 4H); ${ }^{13} \mathrm{C}$ NMR (75 MHz, $\left.\mathrm{CDCl}_{3}\right) \delta 14.4,42.2,74.4,74.8,95.9,125.9,127.2$, 128.0, 146.1; IR (neat) 3543, 3444, 3060, 2982, 2263, 1450, 1170, 1004, $640 \mathrm{~cm}^{-1}$; HRMS (EI) Calcd for $\mathrm{C}_{17} \mathrm{H}_{16} \mathrm{O}_{2}\left(\mathrm{M}^{+}\right)$252.1150. Found 252.1153.

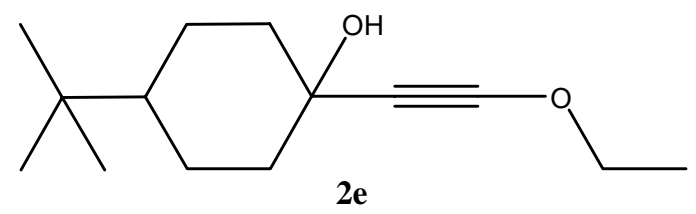

4-tert-butyl-1-ethoxyethynyl-cyclohexanol (2e): white solid; ${ }^{1} \mathrm{H}$ NMR (300 MHz, $\left.\mathrm{CDCl}_{3}\right) \delta 0.87(\mathrm{~s}, 9 \mathrm{H}), 0.98(\mathrm{t} \mathrm{t}, J=11.9,3.5 \mathrm{~Hz}, 1 \mathrm{H}), 1.38(\mathrm{t}, J=7.1 \mathrm{~Hz}, 3 \mathrm{H}), 1.26-1.55$ (m, 4H), 1.71 (br d, $J=12.8 \mathrm{~Hz}, 2 \mathrm{H}), 1.89(\mathrm{~s}, 1 \mathrm{H}), 1.94$ (br d, $J=10.6 \mathrm{~Hz}, 2 \mathrm{H}), 4.09$ (q, $J=7.1 \mathrm{~Hz}, 2 \mathrm{H}) ;{ }^{13} \mathrm{C} \mathrm{NMR}\left(75 \mathrm{MHz}, \mathrm{CDCl}_{3}\right) \delta 14.3,25.0,27.6,32.2,41.1,41.3,47.1$, 69.6, 74.4, 94.0; IR (neat) 3390, 2944, 2865, 2257, 1479, 1444, 1393, 1365, 1178, 1059, 900, $834 \mathrm{~cm}^{-1}$; HRMS (CI) Calcd for $\mathrm{C}_{14} \mathrm{H}_{24} \mathrm{O}_{2}\left(\mathrm{M}+\mathrm{H}^{+}\right)$225.1855. Found 225.1856. 


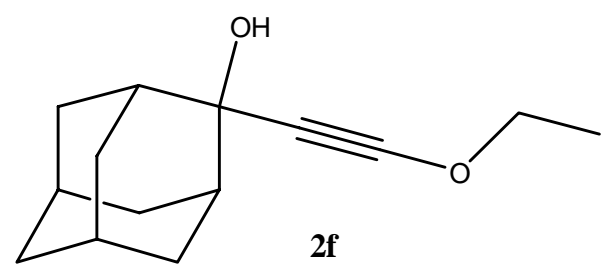

2-ethoxyethynyl-adamantan-2-ol (2f): clear, colorless oil; ${ }^{1} \mathrm{H} \mathrm{NMR}\left(300 \mathrm{MHz}, \mathrm{CDCl}_{3}\right)$ $\delta 1.37(\mathrm{t}, J=7.1 \mathrm{~Hz}, 3 \mathrm{H}), 1.53($ br s, $1 \mathrm{H}), 1.57$ (br s, $1 \mathrm{H}), 1.68-1.80(\mathrm{~m}, 7 \mathrm{H}), 1.88$ (br s, 2H), $2.14($ br t, $J=12.6 \mathrm{~Hz}, 4 \mathrm{H}), 4.09(\mathrm{q}, J=7.1 \mathrm{~Hz}, 2 \mathrm{H}) ;{ }^{13} \mathrm{C} \mathrm{NMR}\left(75 \mathrm{MHz}, \mathrm{CDCl}_{3}\right) \delta$ 14.6, 27.1, 27.3, 32.0, 35.9, 38.0, 39.8, 43.2, 72.7, 74.6, 93.9; IR (neat) 3426, 2902, 2854, 2666, 2259, 1712, 1450, 1247, 1010, 916, $866 \mathrm{~cm}^{-1}$; HRMS (CI) Calcd for $\mathrm{C}_{14} \mathrm{H}_{20} \mathrm{O}_{2}\left(\mathrm{M}+\mathrm{H}^{+}\right)$221.1542. Found 221.1538.

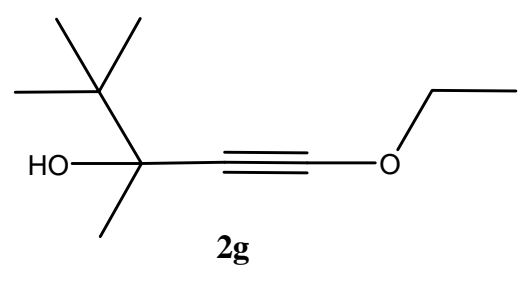

1-ethoxy-3-methyl-3-tert-butyl-1-propyn-3-ol (2g): clear, colorless oil; ${ }^{1} \mathrm{H}$ NMR (300 $\left.\mathrm{MHz}, \mathrm{CDCl}_{3}\right) \delta 1.03(\mathrm{~s}, 9 \mathrm{H}), 1.37(\mathrm{t}, J=7.1 \mathrm{~Hz}, 3 \mathrm{H}), 1.41(\mathrm{~s}, 3 \mathrm{H}), 1.71(\mathrm{~s}, 1 \mathrm{H}), 4.08(\mathrm{q}, J$ $=7.1 \mathrm{~Hz}, 2 \mathrm{H}) ;{ }^{13} \mathrm{C} \mathrm{NMR}\left(75 \mathrm{MHz}, \mathrm{CDCl}_{3}\right) \delta 14.3,25.2,25.6,38.4,41.9,73.9,74.2$, 92.8; IR (neat) 3479, 2971, 2873, 2261, 1481, 1392, 1369, 1219, 1094, 1007, 908, 878 $\mathrm{cm}^{-1}$; HRMS (CI) Calcd for $\mathrm{C}_{10} \mathrm{H}_{19} \mathrm{O}_{2}\left([\mathrm{M}+\mathrm{H}]^{+}\right)$171.1385. Found 171.1390. 


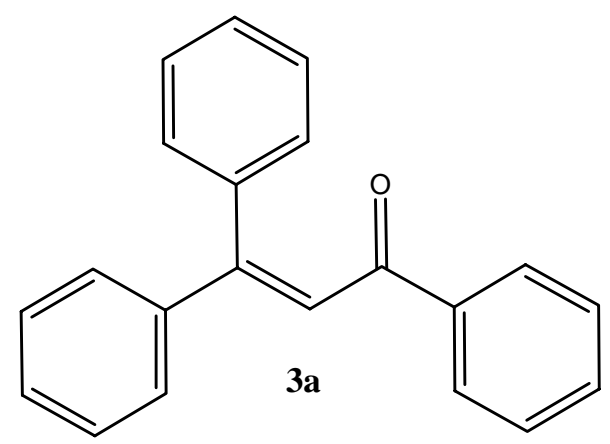

1,3,3-triphenyl-propenone (3a): yellow oil; ${ }^{1} \mathrm{H}$ NMR $\left(300 \mathrm{MHz}, \mathrm{CDCl}_{3}\right) \delta 7.12(\mathrm{~s}, 1 \mathrm{H})$, 7.16-7.21 (m, 2H), 7.25-7.28 (m, 3H), 7.35-7.4 (m, 7H), 7.46-7.51 (m, 1H), $7.91(\mathrm{~d}, J=$ 7.1 Hz, 2H); For full characterization refer to Gurdeep et al. J. Chem. Soc. Perkin Trans. I. 1985, 1289-1294.

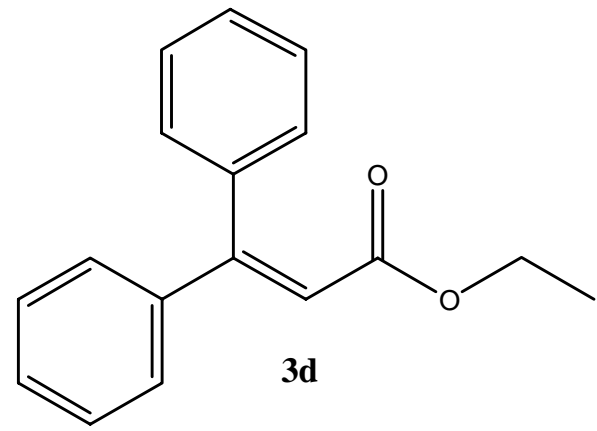

ethyl 3,3-diphenylpropenoate (3d): yellow oil; ${ }^{1} \mathrm{H}$ NMR (300 MHz, $\left.\mathrm{CDCl}_{3}\right) \delta 2.01$ (t, $J$ $=7.1 \mathrm{~Hz}, 3 \mathrm{H}), 4.95(\mathrm{q}, J=7.1 \mathrm{~Hz}, 2 \mathrm{H}), 8.09-8.12(\mathrm{~m}, 2 \mathrm{H}), 8.18-8.30(\mathrm{~m}, 8 \mathrm{H})$; For full characterization refer toLe Tadic-Biadatti et al. J. Org. Chem. 1997, 62, 559-563 and Rathke, M. et al. Synth. Commun. 1990, 20, 869-875. 


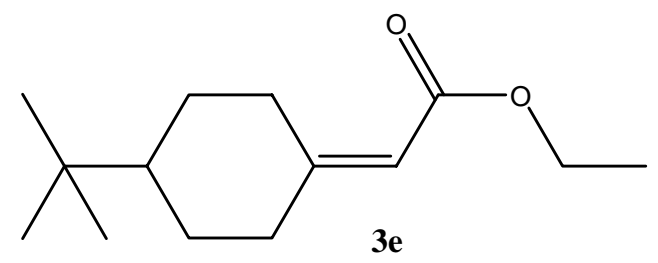

4-tert-butylcyclohexylidene-acetic acid ethyl ester (3e): clear, colorless oil; ${ }^{1} \mathrm{H}$ NMR $\left(300 \mathrm{MHz}, \mathrm{CDCl}_{3}\right) \delta 0.84(\mathrm{~s}, 9 \mathrm{H}), 1.04-1.28(\mathrm{~m}, 3 \mathrm{H}), 1.26(\mathrm{t}, J=7.1 \mathrm{~Hz}, 3 \mathrm{H}), 1.76-1.95$ $(\mathrm{m}, 3 \mathrm{H}), 2.14(\mathrm{t} \mathrm{d}, J=13.5,3.9 \mathrm{~Hz}, 1 \mathrm{H}), 2.27-2.32(\mathrm{~m}, 1 \mathrm{H}), 3.86(\mathrm{~d} \mathrm{q}, J=13.7,2.7,1 \mathrm{H})$, $4.12(\mathrm{q}, J=7.1 \mathrm{~Hz}, 2 \mathrm{H}), 5.58(\mathrm{~s}, 1 \mathrm{H}) ;{ }^{13} \mathrm{C} \mathrm{NMR}\left(75 \mathrm{MHz}, \mathrm{CDCl}_{3}\right) \delta 14.3,27.5,28.4$, 29.2, 29.5, 32.4, 37.9, 47.8, 59.4, 112.7, 163.5, 166.9; IR (neat) 3412, 2948, 2867, 1716, 1648, 1478, 1365, 1247, 1143, 1041, $862 \mathrm{~cm}^{-1}$; HRMS (CI) Calcd for $\mathrm{C}_{14} \mathrm{H}_{24} \mathrm{O}_{2}\left(\mathrm{M}+\mathrm{H}^{+}\right)$ 225.1855. Found 225.1849.

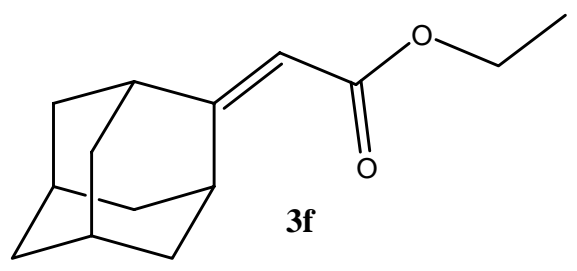

adamantan-2-ylidene-acetic acid ethyl ester (3f): clear, colorless oil: ${ }^{1} \mathrm{H}$ NMR (300 $\left.\mathrm{MHz}, \mathrm{CDCl}_{3}\right) \delta 1.27(\mathrm{t}, J=7.1 \mathrm{~Hz}, 3 \mathrm{H}), 1.86($ br s, 6H), 1.93-1.96 (m, 6H), 2.43 (br s, 1H), 4.07 (br s, 1H), 4.13 (q, $J=7.1 \mathrm{~Hz}, 2 \mathrm{H}), 5.58(\mathrm{~s}, 1 \mathrm{H})$; For full characterization refer to L. Griaud et al. Tetrahedron, 1998, 54, 11899-11906. 


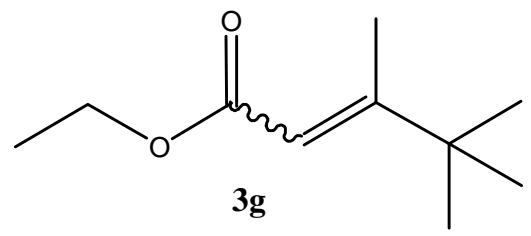

(E/Z)-3,4,4-trimethyl-pent-2-enoic acid ethyl ester (3g): clear, colorless oil: ${ }^{1} \mathrm{H}$ NMR (300 MHz, $\mathrm{CDCl}_{3}, \mathrm{E}$ isomer) $\delta 1.10(\mathrm{~s}, 9 \mathrm{H}), 1.28(\mathrm{t}, J=7.1 \mathrm{~Hz}, 3 \mathrm{H}), 2.16(\mathrm{br} \mathrm{d}, J=1.1$ $\mathrm{Hz}, 3 \mathrm{H}), 4.14(\mathrm{q}, J=7.1 \mathrm{~Hz}, 2 \mathrm{H}), 5.74(\mathrm{q}, J=1.1 \mathrm{~Hz}, 1 \mathrm{H}) ;{ }^{1} \mathrm{H}$ NMR $\left(300 \mathrm{MHz}, \mathrm{CDCl}_{3}\right.$, $\mathrm{Z}$ isomer) $\delta 1.20(\mathrm{~s}, 9 \mathrm{H}), 1.28(\mathrm{t}, J=7.1 \mathrm{~Hz}, 3 \mathrm{H}), 1.84(\mathrm{br} \mathrm{d}, J=1.3 \mathrm{~Hz}, 3 \mathrm{H}), 4.14(\mathrm{q}, J=$ $7.1 \mathrm{~Hz}, 2 \mathrm{H}), 5.63(\mathrm{q}, J=1.3 \mathrm{~Hz}, 1 \mathrm{H}) ;{ }^{13} \mathrm{C}$ NMR $\left(75 \mathrm{MHz}, \mathrm{CDCl}_{3}, \mathrm{E} / \mathrm{Z}\right.$ mixture $) \delta 14.1$, $14.3,15.1,23.9,28.5,29.0,36.4,37.9,59.4,60.0,112.9,116.6,158.5,167.2,167.5$, 167.9; IR (neat, E/Z mixture) 2970, 2873, 1719, 1634, 1466, 1372, 1262, 1182, 1123, 1054, $868 \mathrm{~cm}^{-1}$; HRMS (EI) Calcd for $\mathrm{C}_{10} \mathrm{H}_{18} \mathrm{O}_{2}\left(\mathrm{M}^{+}\right)$170.1307. Found 170.1306.

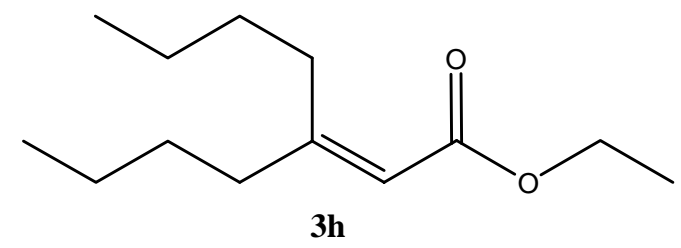

3-butyl-hept-2-enoic acid ethyl ester (3h): clear, colorless oil: ${ }^{1} \mathrm{H}$ NMR $(300 \mathrm{MHz}$, $\left.\mathrm{CDCl}_{3}\right) \delta$ 0.89-0.94 (m, 6H), $1.27(\mathrm{t}, J=7.1 \mathrm{~Hz}, 3 \mathrm{H}), 1.28-1.47(\mathrm{~m}, 8 \mathrm{H}), 2.13$ (br t, $J=$ $7.6 \mathrm{~Hz}, 2 \mathrm{H}), 2.59$ (br t $J=7.6 \mathrm{~Hz}, 2 \mathrm{H}), 4.13(\mathrm{q}, J=7.1 \mathrm{~Hz}, 2 \mathrm{H}), 5.61(\mathrm{~s}, 1 \mathrm{H}),{ }^{13} \mathrm{C} \mathrm{NMR}$ $\left(75 \mathrm{MHz}, \mathrm{CDCl}_{3}\right) \delta 13.88,13.92,14.3,22.4,23.0,29.8,30.8,31.9,38.1,59.3,115.0$, 164.8, 166.6; IR (neat) 2958, 2931, 2872, 1716, 1642, 1466, 1378, 1190, 1147, 1039, 862 $\mathrm{cm}^{-1}$; HRMS (CI) Calcd for $\mathrm{C}_{13} \mathrm{H}_{24} \mathrm{O}_{2}\left(\mathrm{M}+\mathrm{H}^{+}\right)$213.1855. Found 213.1857. 


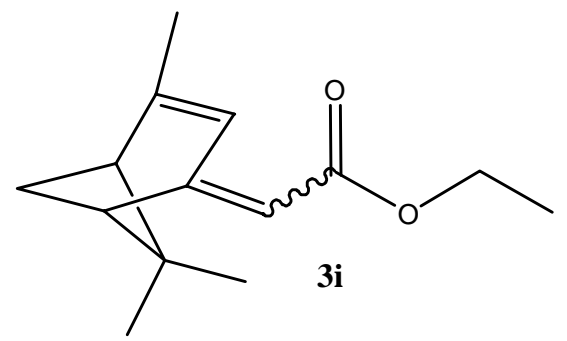

(4,6,6-trimethyl-bicyclo[3.1.1]hept-3-en-(2E/Z)-ylidene)-acetic acid ester (3i): clear oil; ${ }^{1} \mathrm{H}$ NMR $\left(300 \mathrm{MHz}, \mathrm{CDCl}_{3}, \mathrm{E}\right.$ isomer) $\delta 0.86(\mathrm{~s}, 3 \mathrm{H}), 1.28(\mathrm{t}, J=7.1 \mathrm{~Hz}, 3 \mathrm{H}), 1.40$ (s, 3H), $1.68(\mathrm{~d}, J=7.9 \mathrm{~Hz}, 1 \mathrm{H}), 1.90(\mathrm{~d}, J=1.5 \mathrm{~Hz}, 3 \mathrm{H}), 2.20-2.45(\mathrm{~m}, 1 \mathrm{H}), 2.53-2.63$ (m, 2H), 4.08-4.20 (m, 2H), $5.32(\mathrm{~s}, 1 \mathrm{H}), 7.13(\mathrm{~s}, 1 \mathrm{H}) ;{ }^{1} \mathrm{H}$ NMR (300 MHz, $\mathrm{CDCl}_{3}, \mathrm{Z}$ isomer) $\delta 0.84(\mathrm{~s}, 3 \mathrm{H}), 1.26(\mathrm{t}, J=7.1 \mathrm{~Hz}, 3 \mathrm{H}), 1.44(\mathrm{~s}, 3 \mathrm{H}), 1.58(\mathrm{~d}, J=8.8 \mathrm{~Hz}, 1 \mathrm{H})$, $1.86(\mathrm{~d}, J=1.4,3 \mathrm{H}), 2.20-2.45(\mathrm{~m}, 1 \mathrm{H}), 2.53-2.63(\mathrm{~m}, 2 \mathrm{H}), 4.08-4.20(\mathrm{~m}, 2 \mathrm{H}), 5.46(\mathrm{~s}$ 1H), $5.77(\mathrm{~s}, 1 \mathrm{H}) ;{ }^{13} \mathrm{C} \mathrm{NMR}\left(75 \mathrm{MHz}, \mathrm{CDCl}_{3}, \mathrm{E} / \mathrm{Z}\right.$ mixture) $\delta 14.3,14.4,21.7,21.8,23.2$, 23.6, 26.4, 26.5, 37.5, 38.1, 45.3, 47.8, 48.2, 49.0, 49.1, 53.1, 59.2, 59.3, 107.6, 110.0, 117.6, 121.6, 156.9, 158.0, 159.6, 161.2, 166.8, 167.4; IR (neat) 2979, 2930, 2870, 1708, 1622, 1466, 1443, 1380, 1370, 1226, 1164, 1040, 874, $705 \mathrm{~cm}^{-1}$; HRMS (EI) Calcd for $\mathrm{C}_{14} \mathrm{H}_{20} \mathrm{O}_{2}\left(\mathrm{M}^{+}\right)$220.1463. Found 220.1462. 


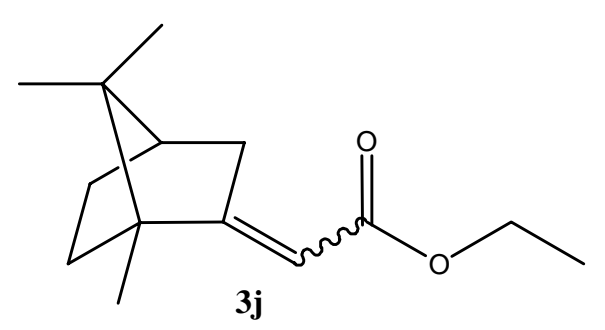

(1,7,7-trimethyl-bicyclo[2.2.1]hept-(2E/Z)-ylidene)-acetic acid ethyl ester (3J): clear, colorless oil; ${ }^{1} \mathrm{H}$ NMR (300 MHz, $\mathrm{CDCl}_{3}$ ) diagnostic resonance signals for $\mathrm{E}$ and $\mathrm{Z}$ isomers appeared at $\delta 5.58(\mathrm{brt}, J=2.3 \mathrm{~Hz}, 1 \mathrm{H}), 5.64(\mathrm{brt}, J=2.0 \mathrm{hz}, 1 \mathrm{H}) ;{ }^{13} \mathrm{C} \mathrm{NMR}$

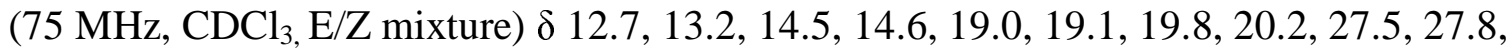
34.0, 34.4, 38.6, 40.6, 44.0, 44.6, 48.2, 50.0, 53.9, 54.0, 59.6, 60.0, 108.2, 112.2, 166.9, 167.6, 175.6; IR (neat, E/Z mixture) 2955, 2874, 1714, 1652, 1450, 1368, 1306, 1288, $1200,1177,1097,1048,873,850 \mathrm{~cm}^{-1}$; HRMS (CI) Calcd for $\mathrm{C}_{14} \mathrm{H}_{22} \mathrm{O}_{2}\left(\mathrm{M}+\mathrm{H}^{+}\right)$ 223.1698. Found 223.1695.

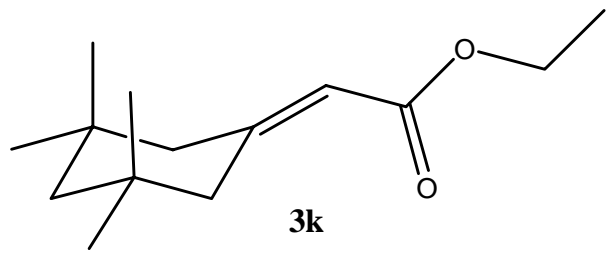

(3,3,5,5-tetramethyl-cyclohexylidene)-acetic acid ethyl ester (3k): clear, colorless oil; ${ }^{1} \mathrm{H}$ NMR (300 MHz, $\left.\mathrm{CDCl}_{3}\right) \delta 0.95(\mathrm{~s}, 6 \mathrm{H}), 0.97(\mathrm{~s}, 6 \mathrm{H}), 1.28(\mathrm{t}, J=7.1 \mathrm{~Hz}, 3 \mathrm{H}), 1.33(\mathrm{~s}$, 2H), 1.95 (s, 2H), 2.61 (s, 2H), 4.15 (q, $J=7.1 \mathrm{~Hz}, 2 \mathrm{H}), 5.69(\mathrm{~s}, 1 \mathrm{H}) ;{ }^{13} \mathrm{C} \mathrm{NMR}(75 \mathrm{MHz}$, $\left.\mathrm{CDCl}_{3}\right) \delta 14.3,30.8,30.9,34.8,34.9,42.2,51.1,52.5,59.4,115.8,160.4,166.6$; IR (neat) $2953,2896,1716,1646,1461,1376,1229,1154,1043,862 \mathrm{~cm}^{-1}$; HRMS (EI) Calcd for $\mathrm{C}_{14} \mathrm{H}_{24} \mathrm{O}_{2}\left(\mathrm{M}^{+}\right)$224.1776. Found 224.1771. 


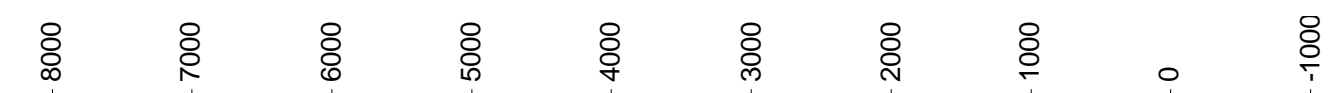

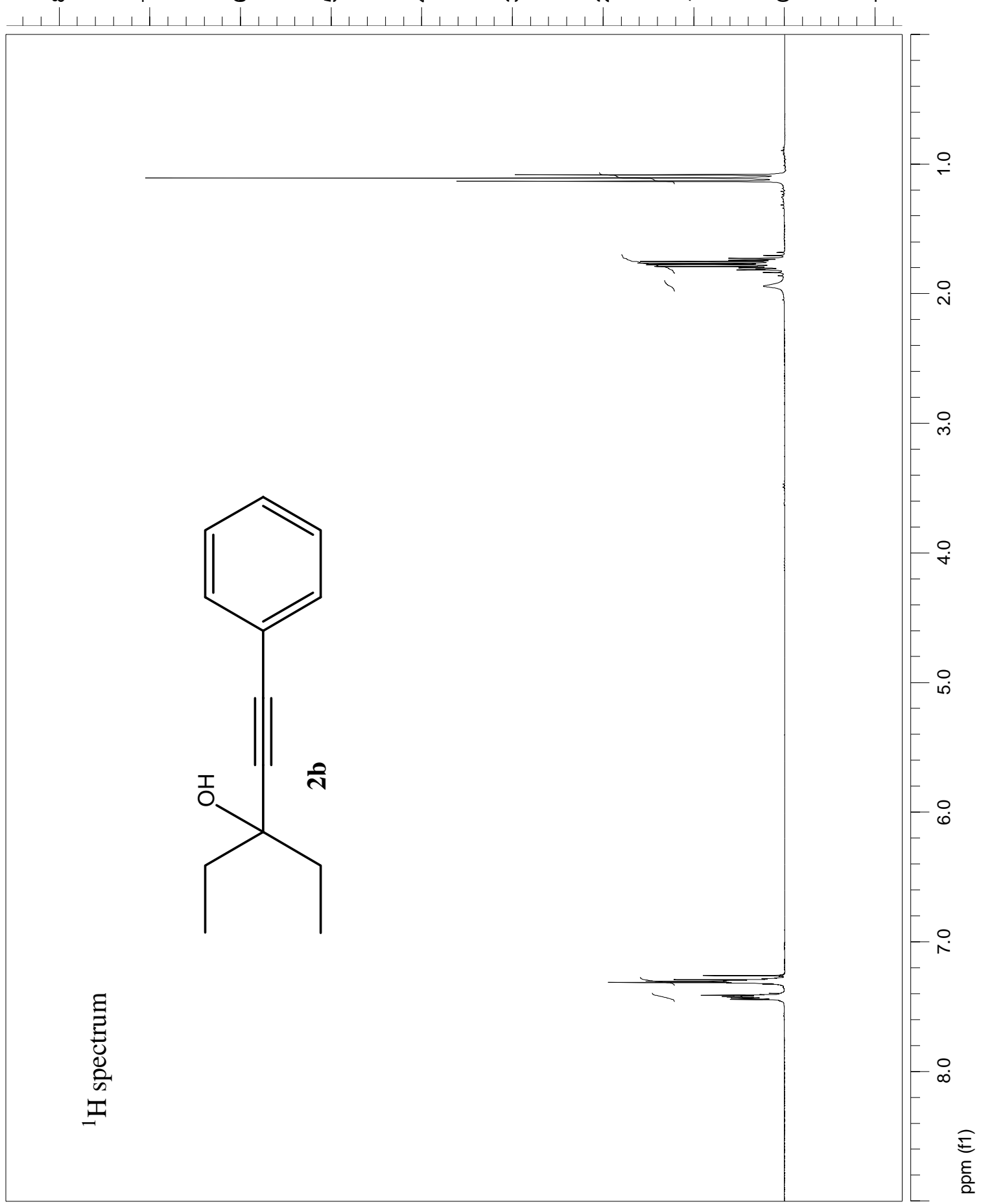




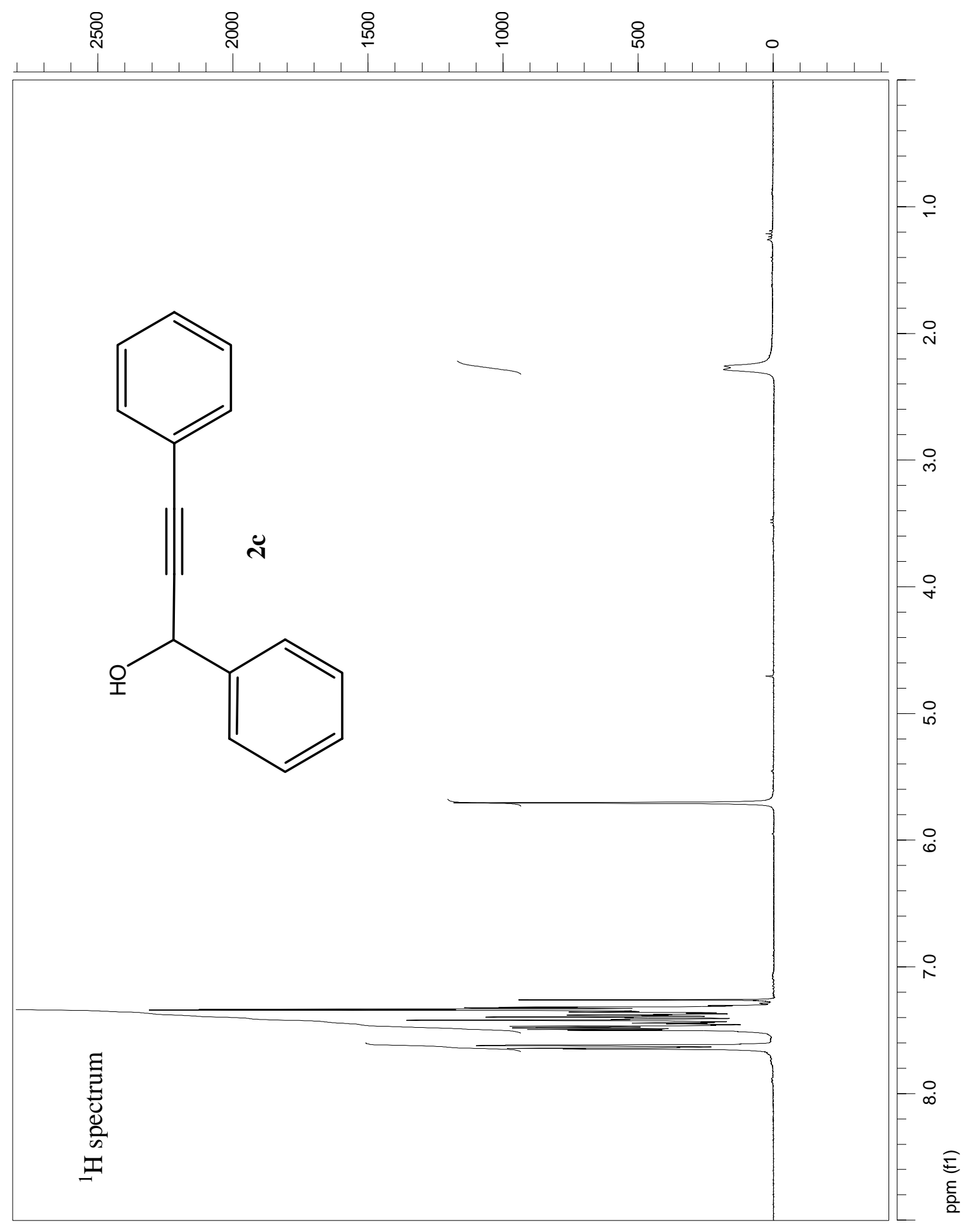

U 


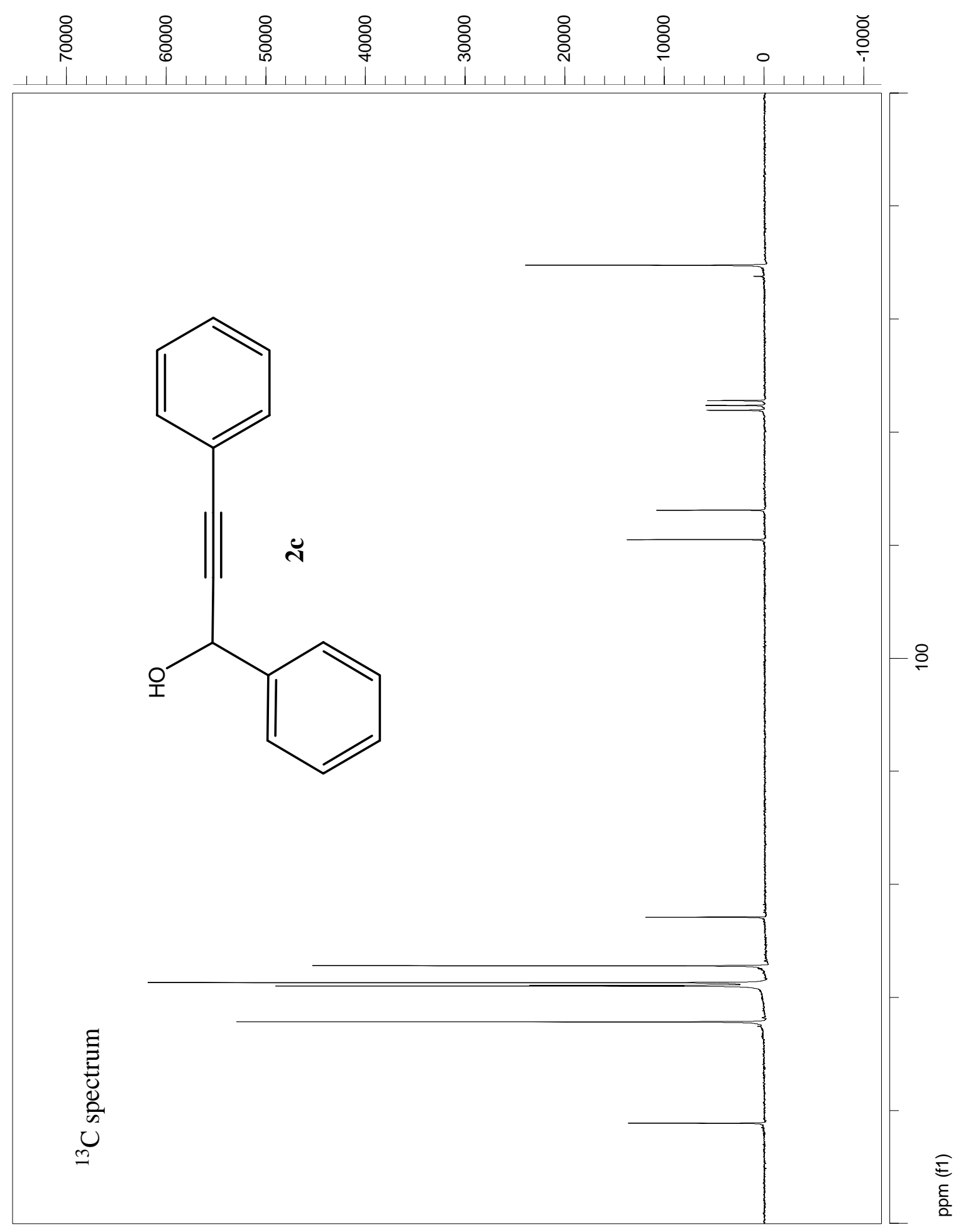

$\frac{n}{n}$

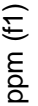




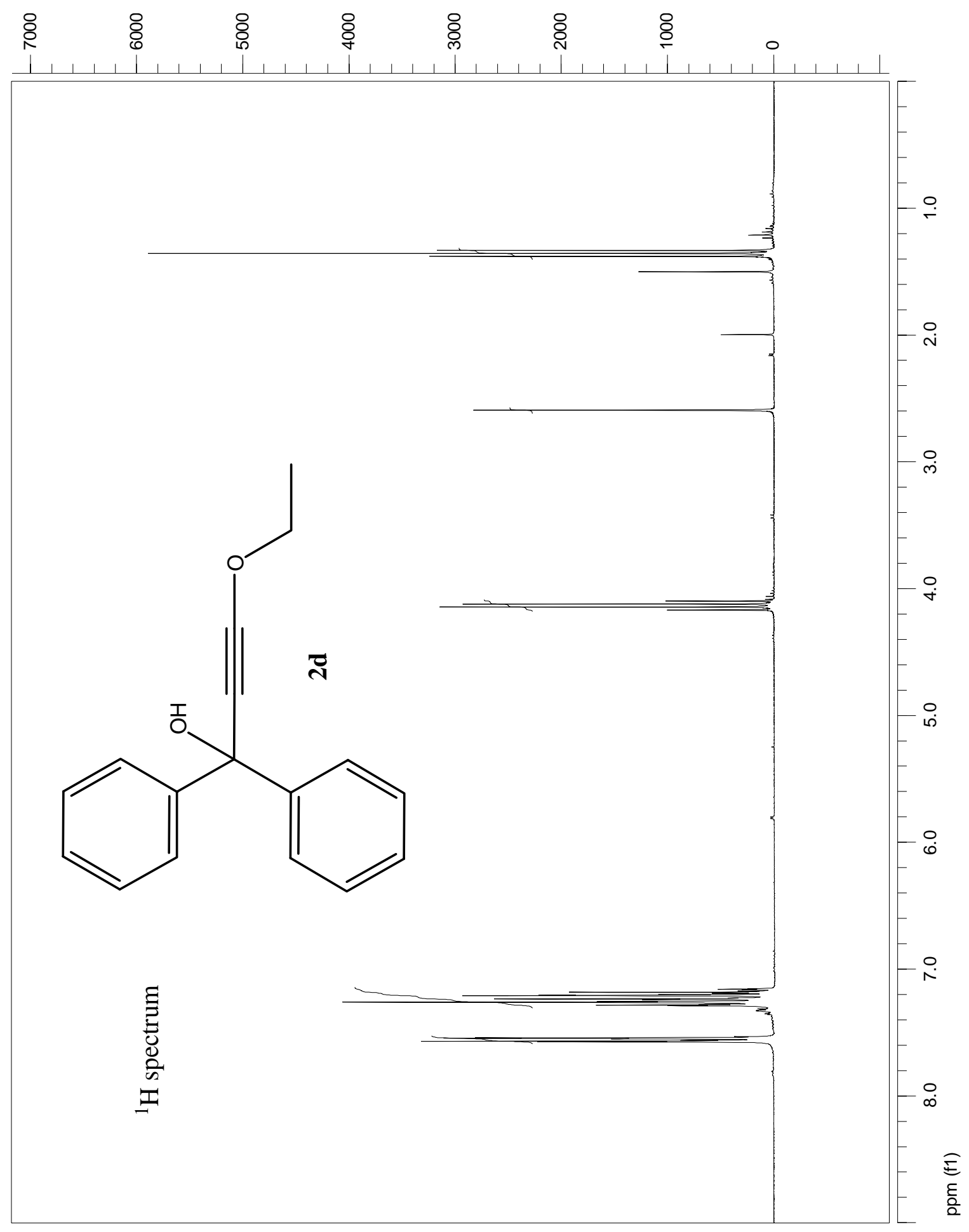




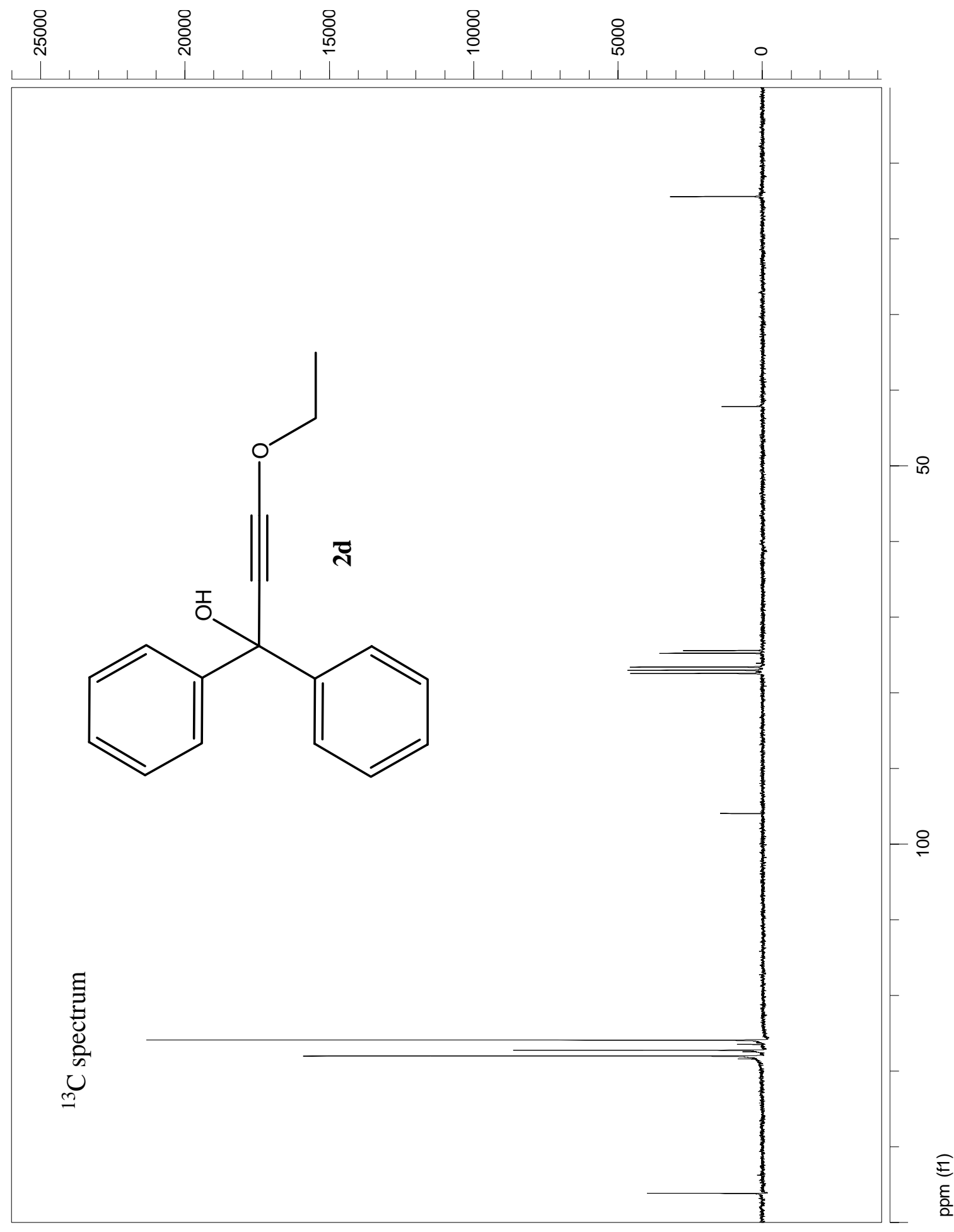

$\frac{N}{n}$ 


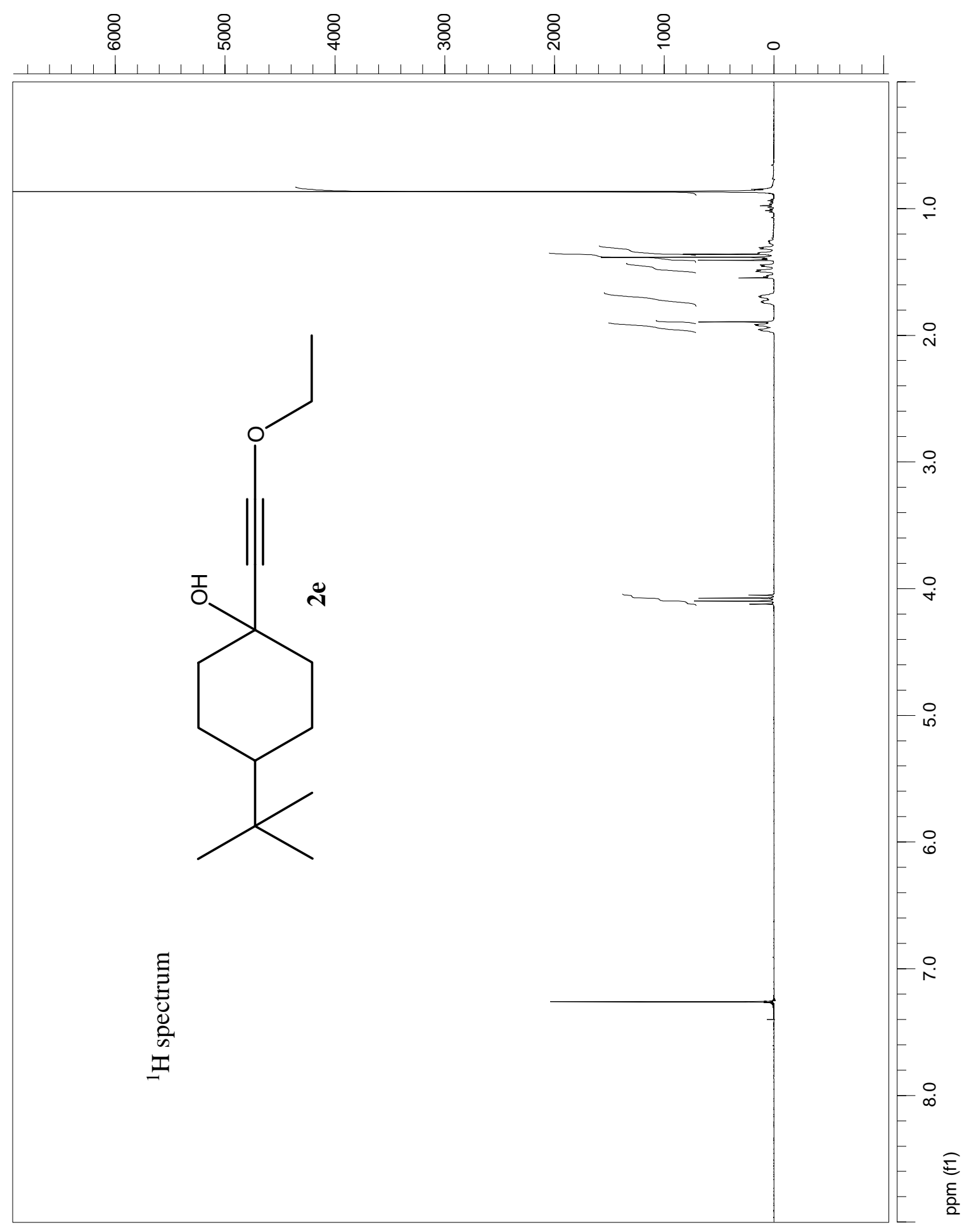




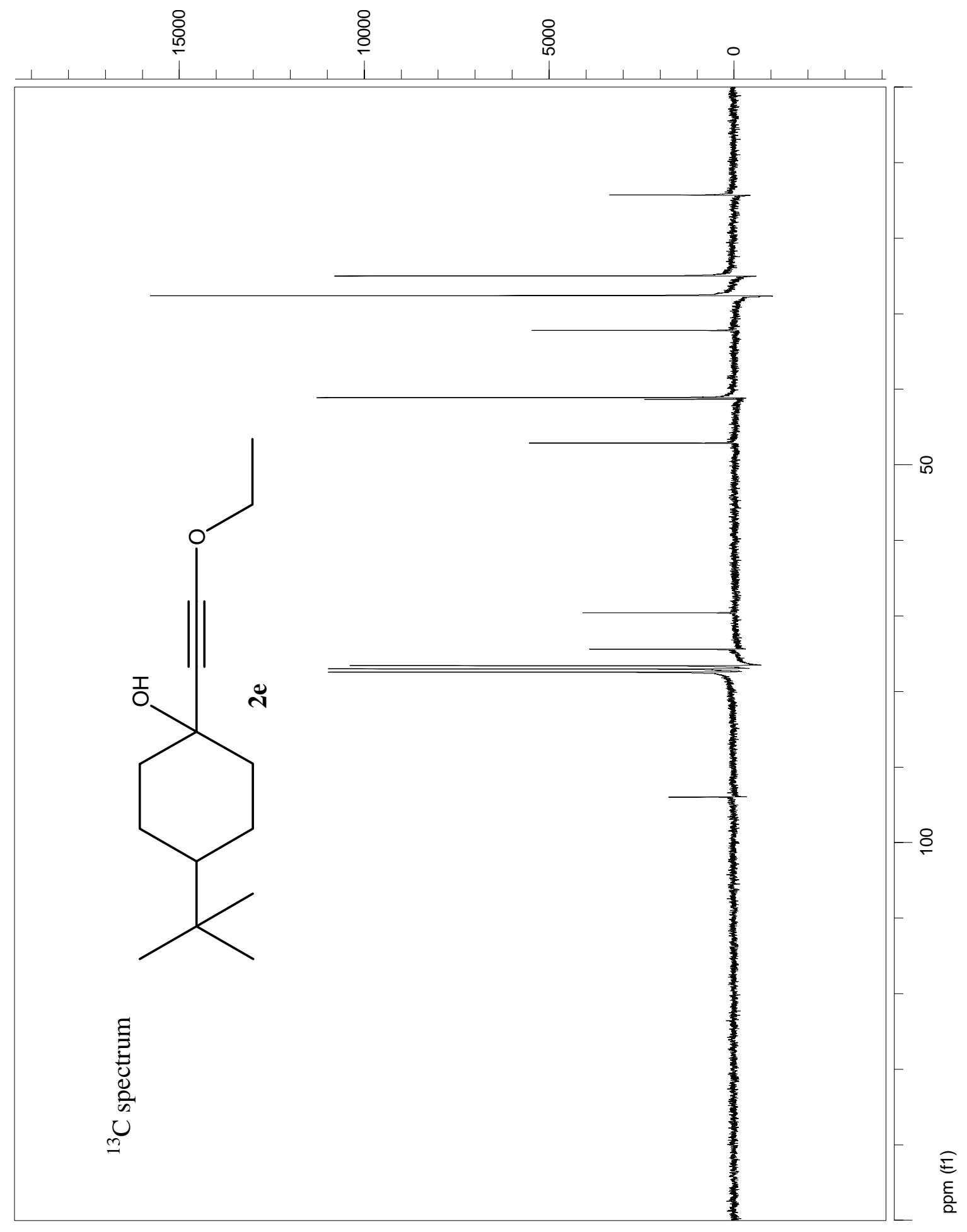




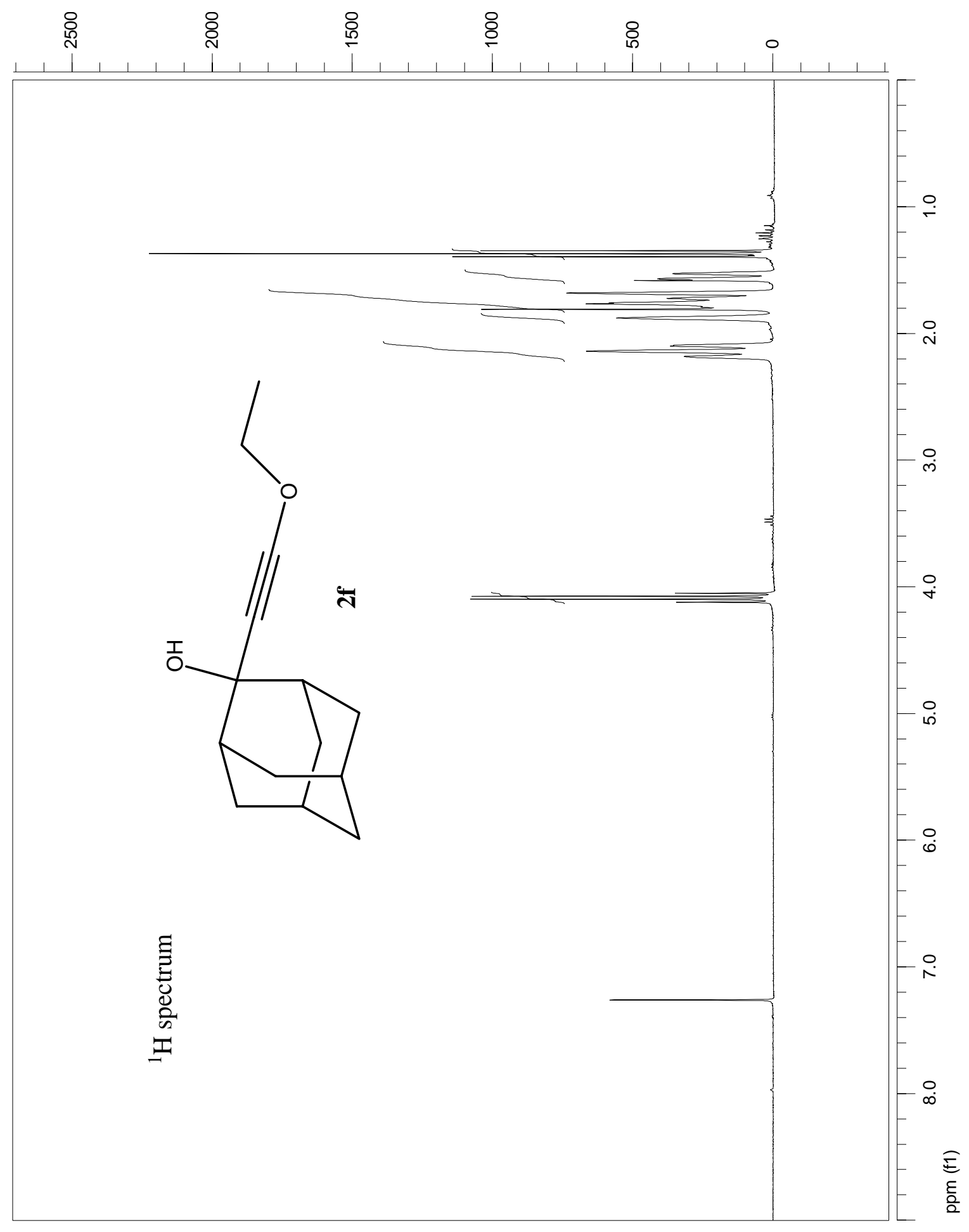

๑ั 


$$
\text { 南 }
$$




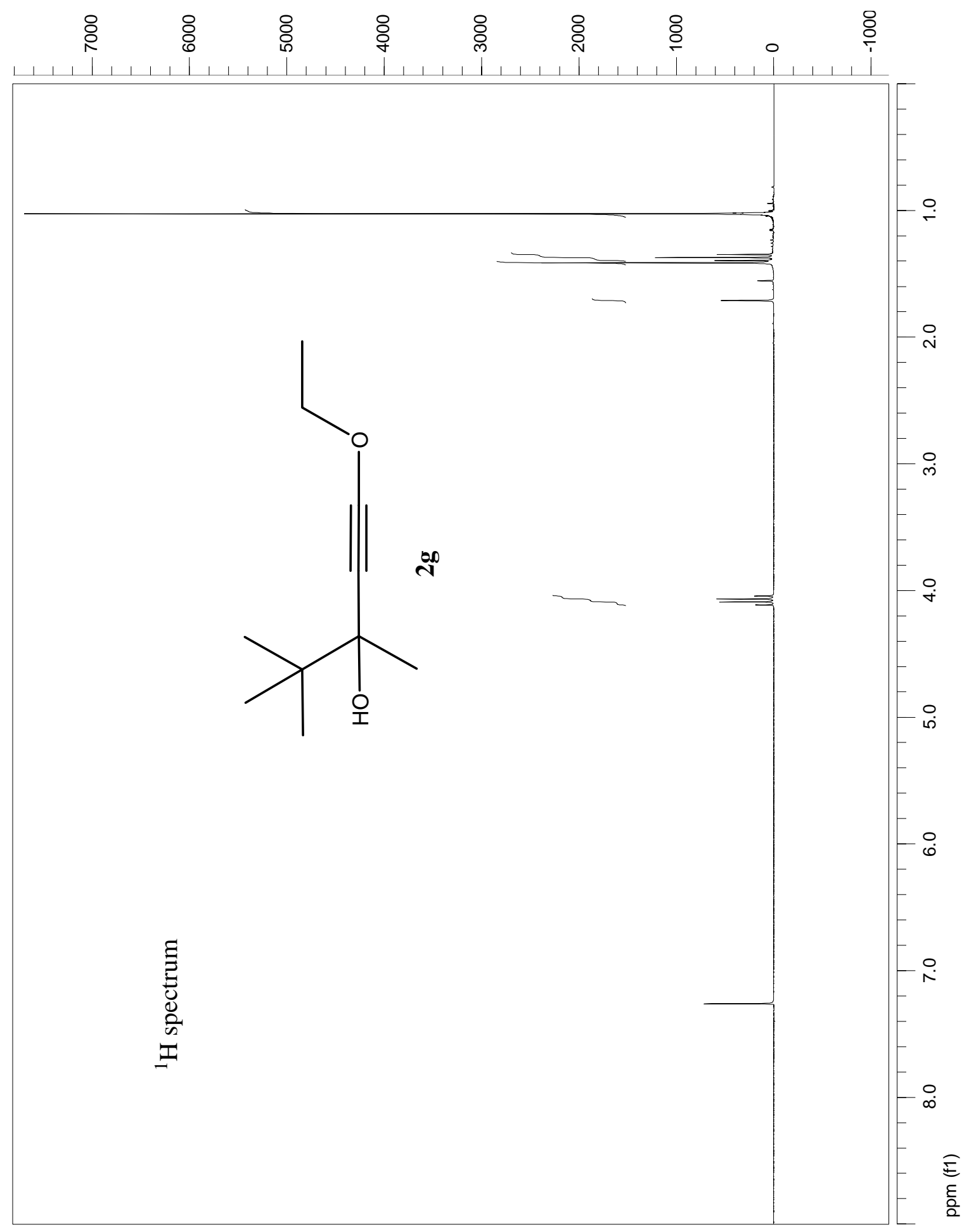




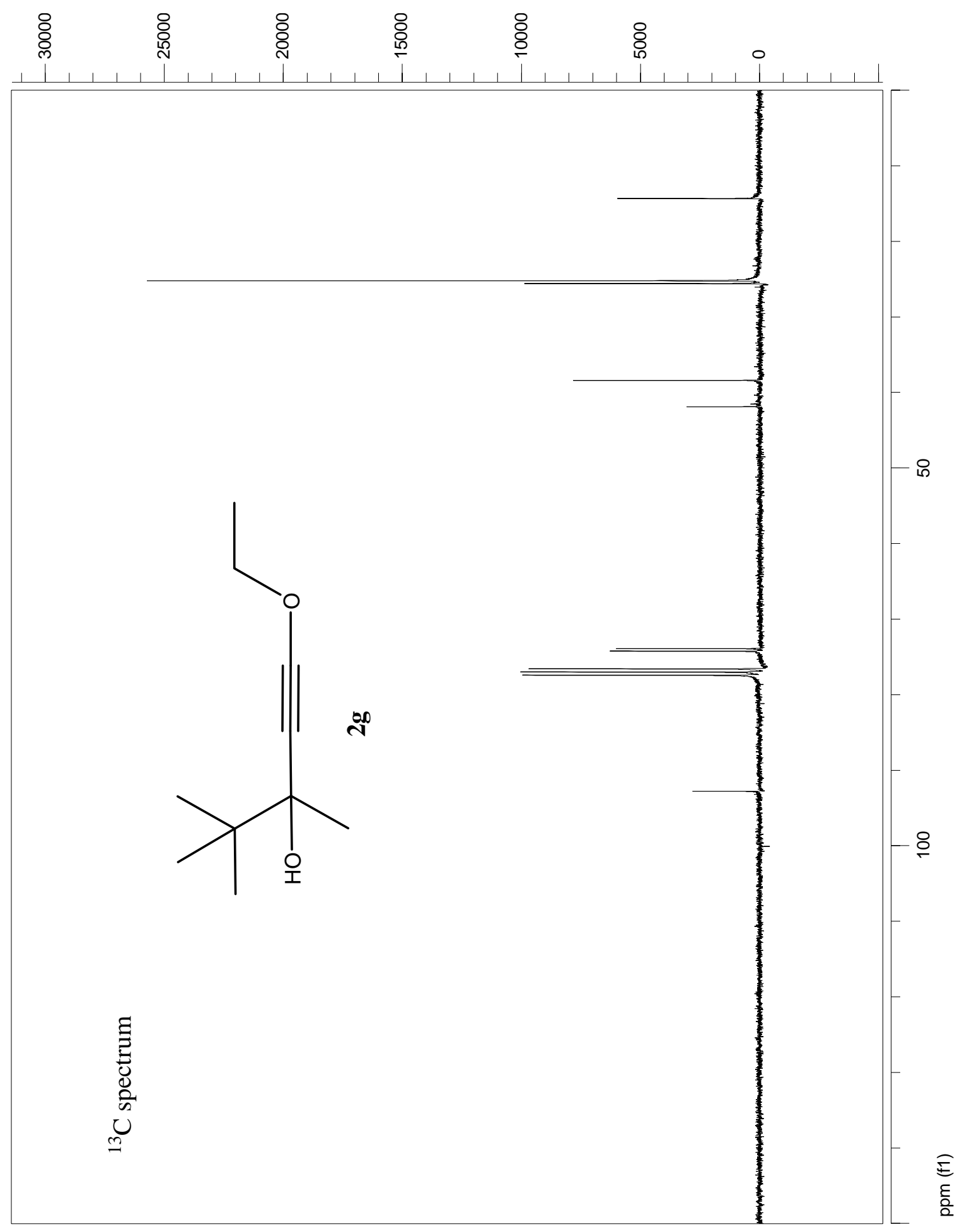

ชิ 


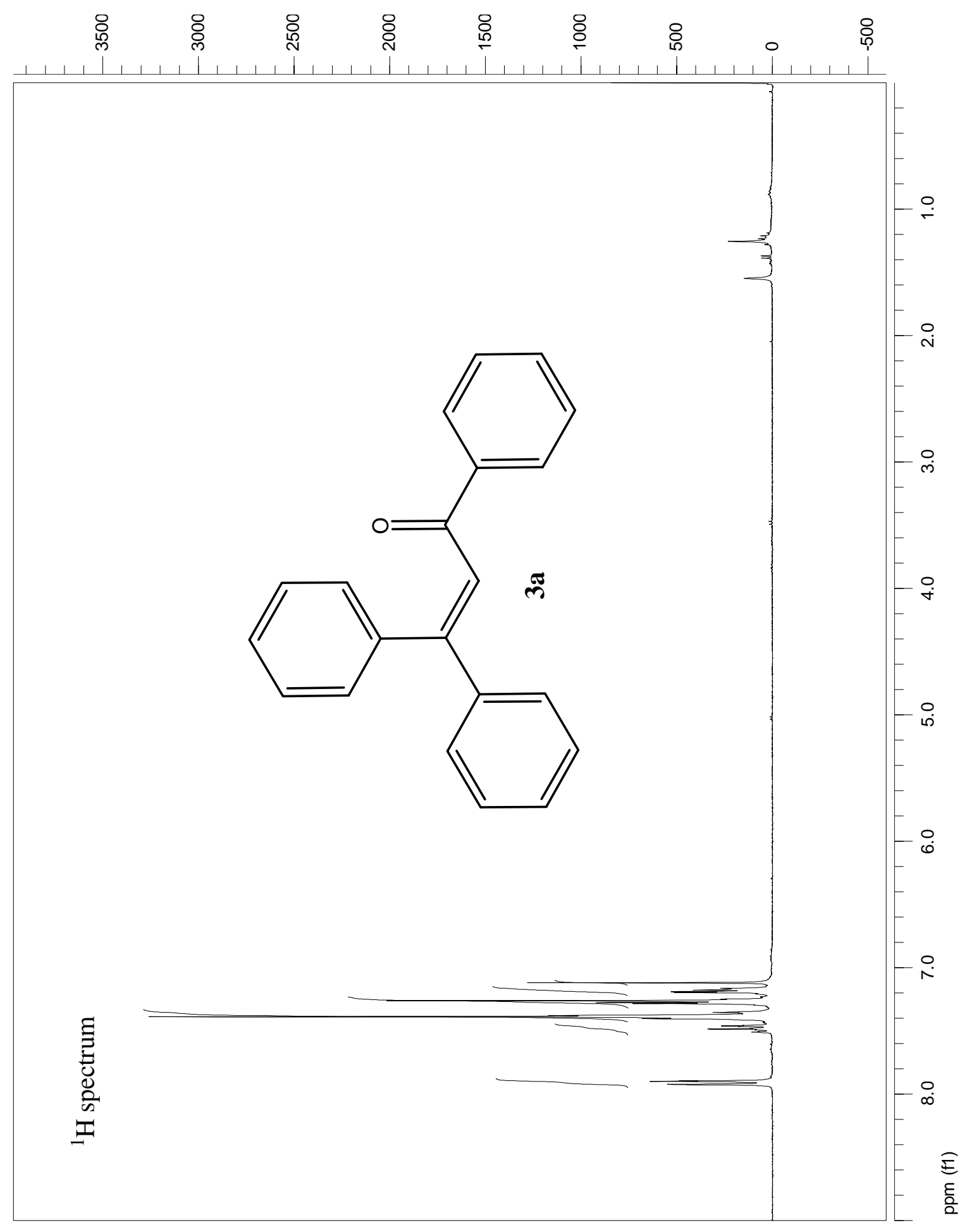




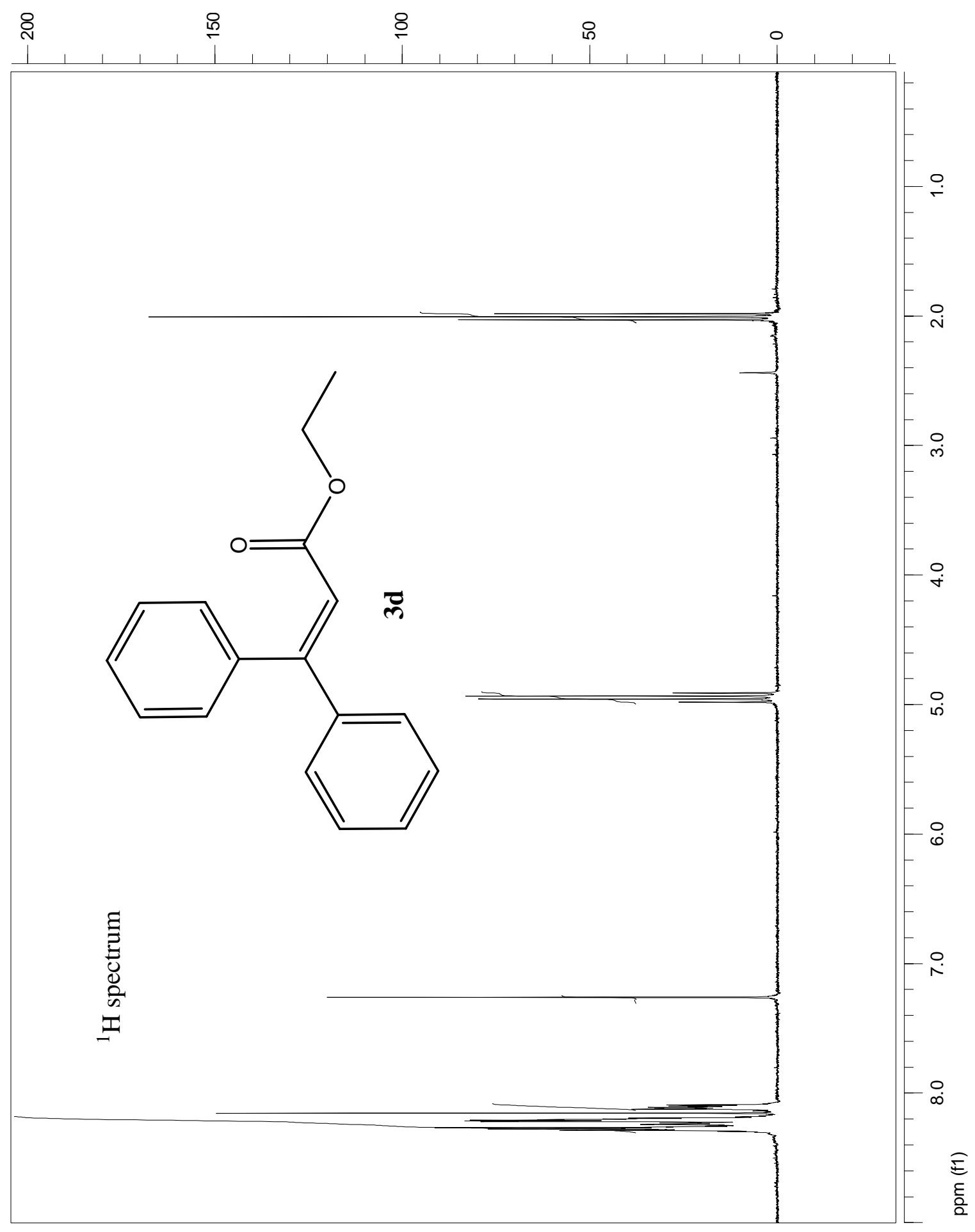




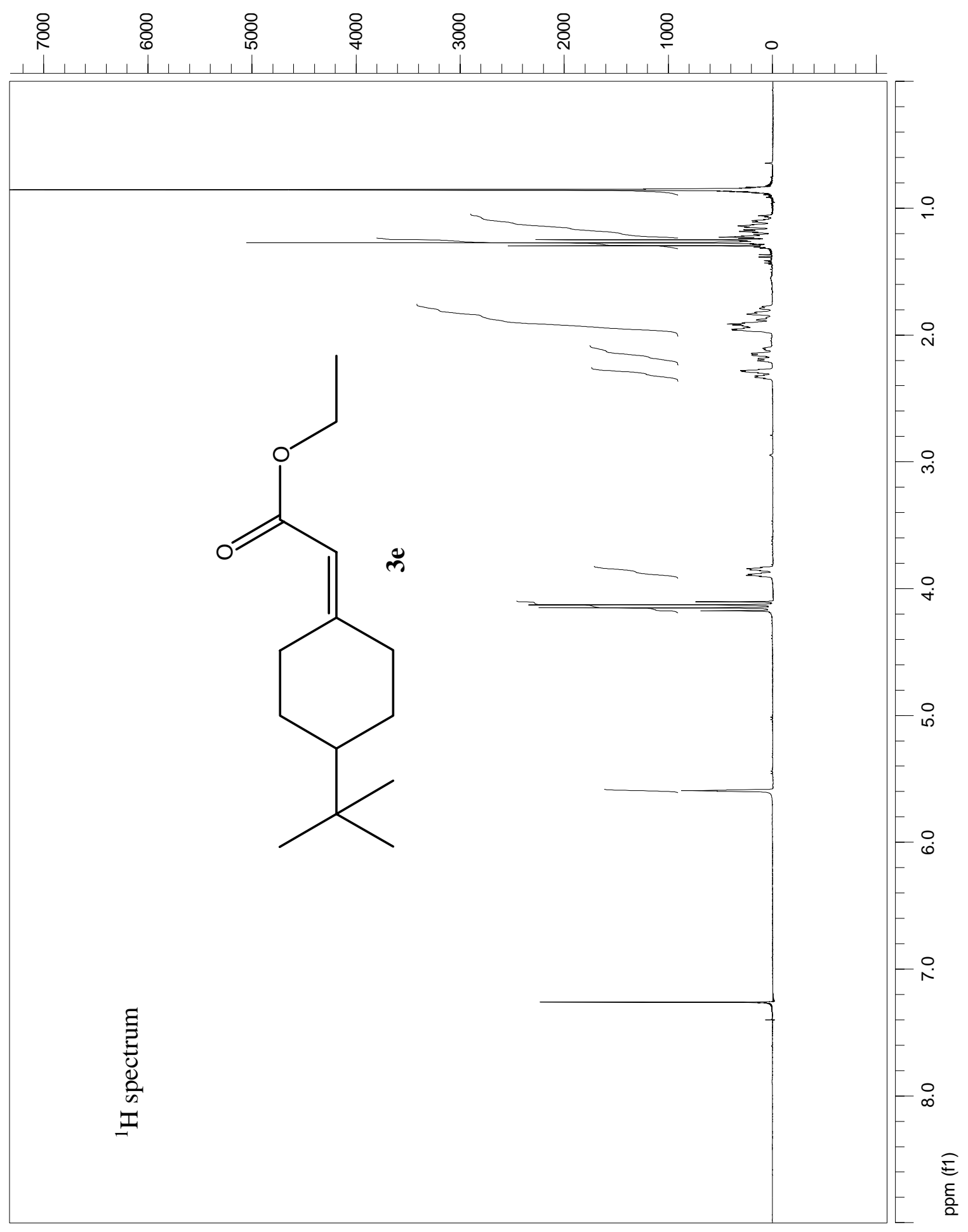




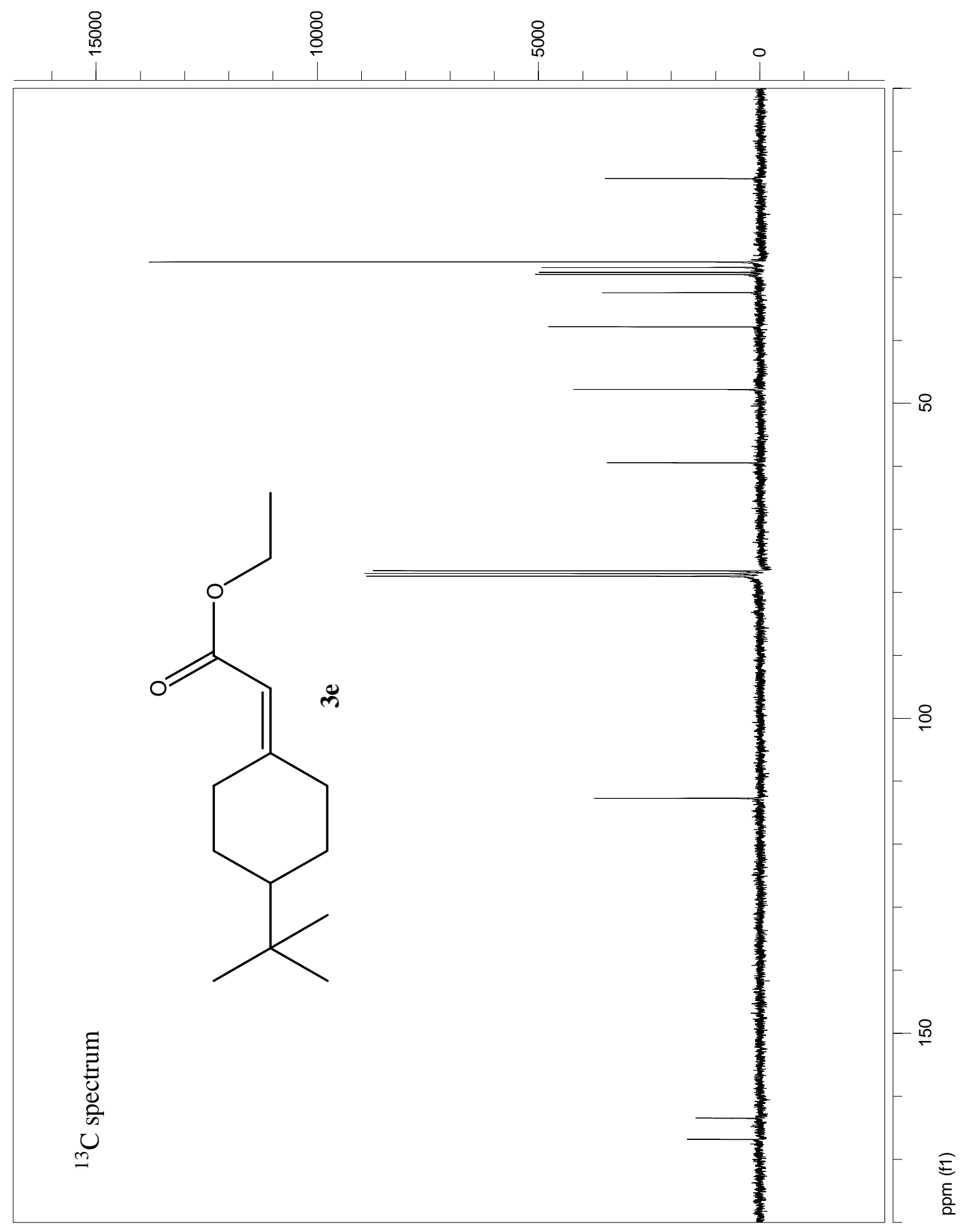

สิ 


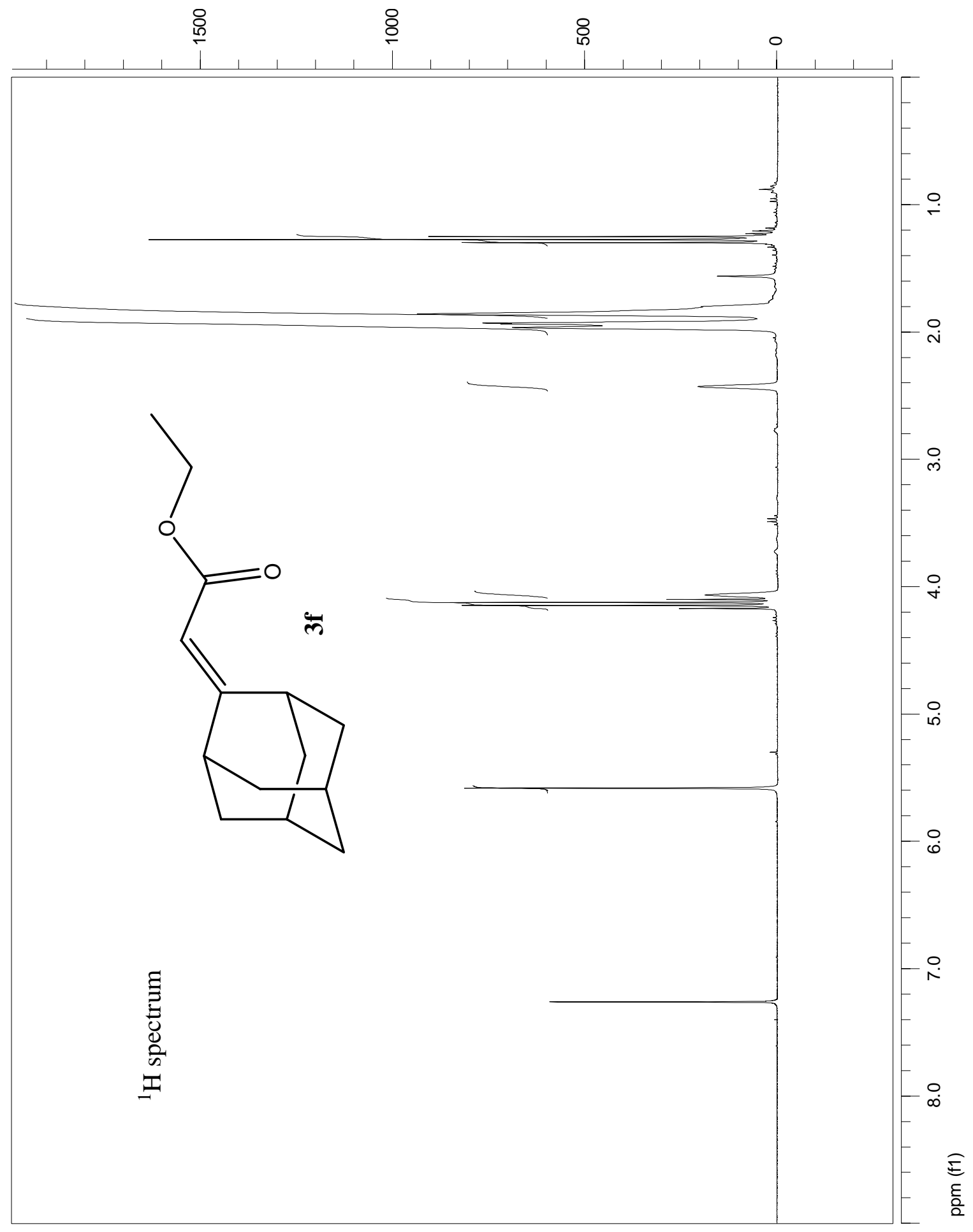




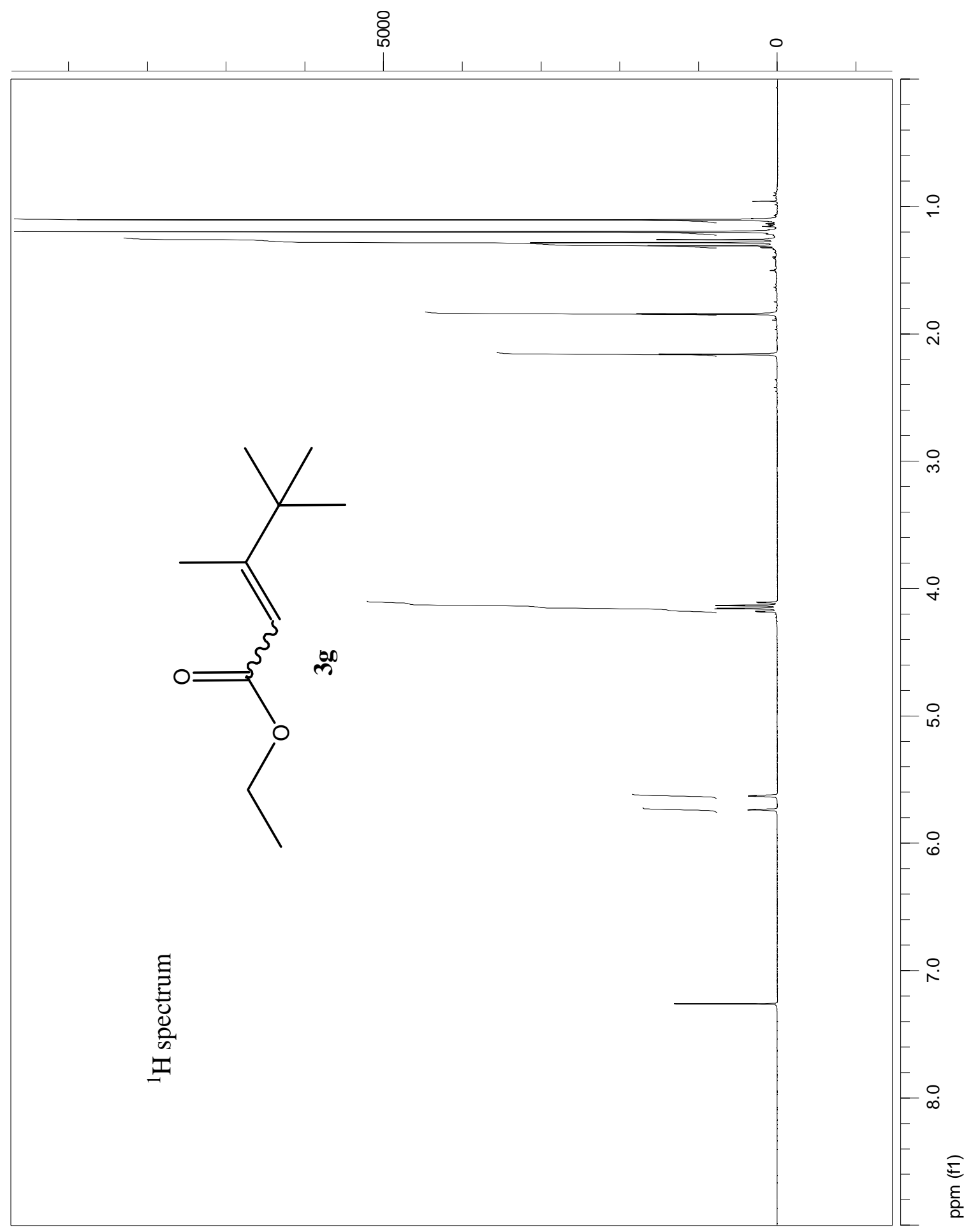

สิ 


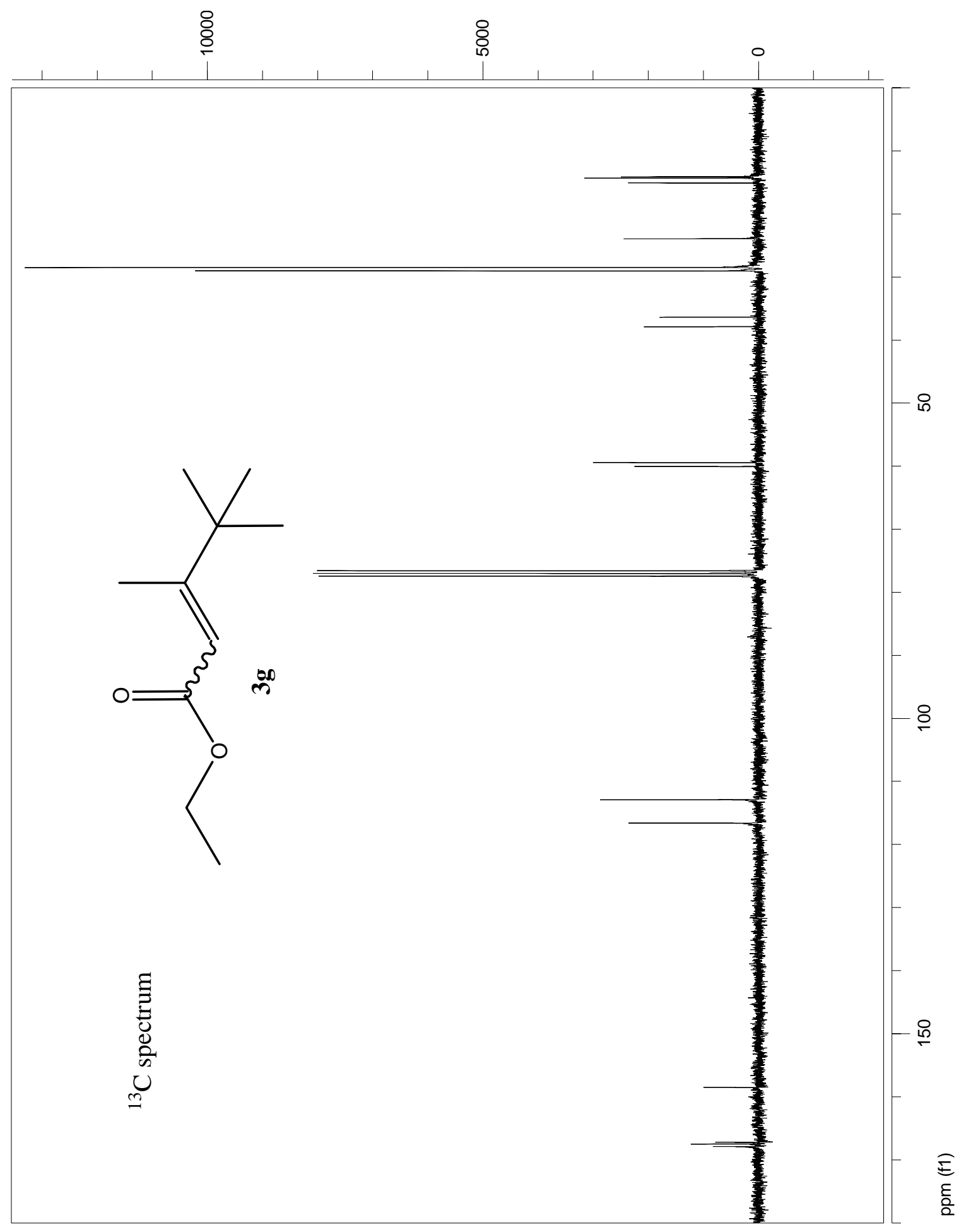




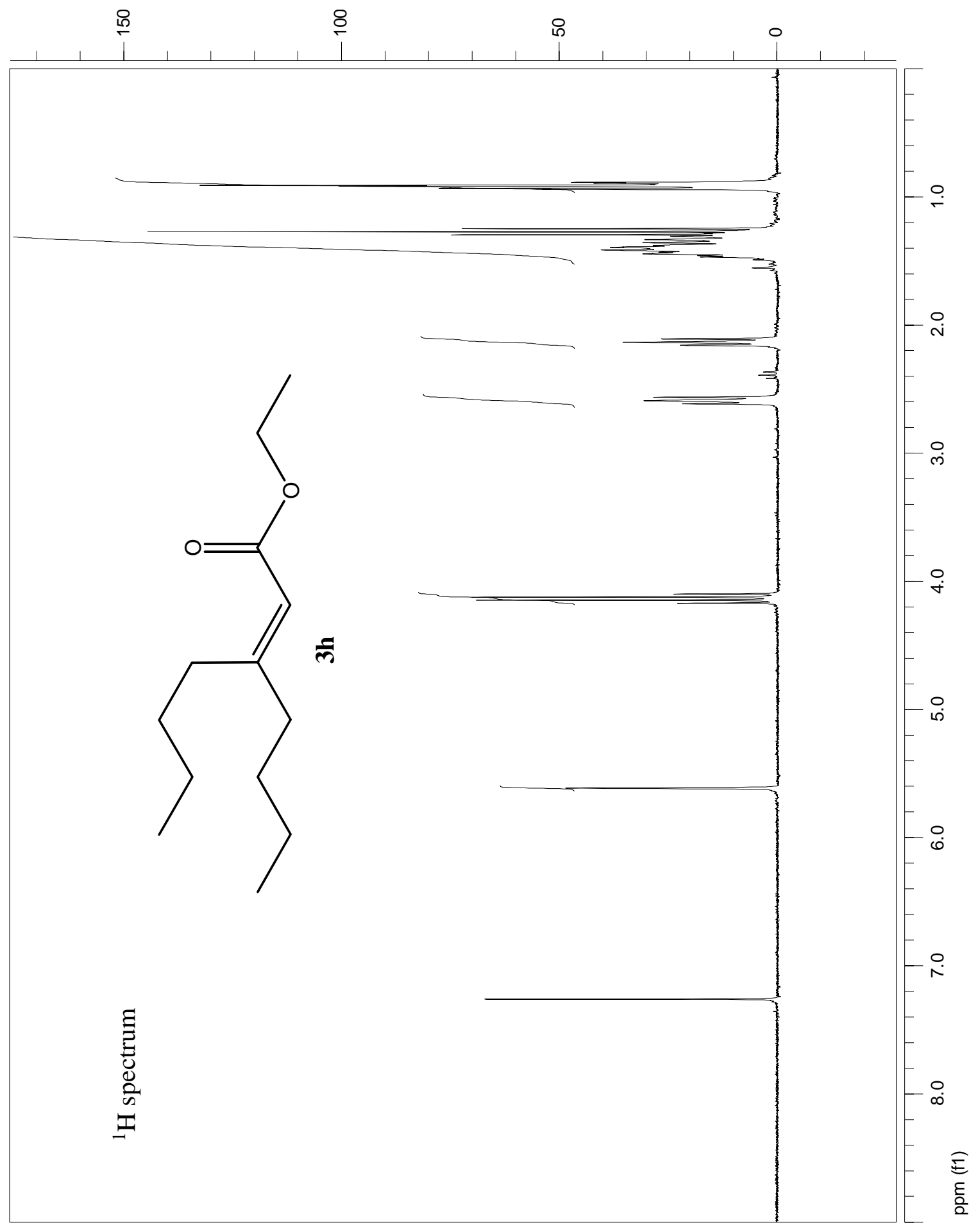

$\bar{n}$ 


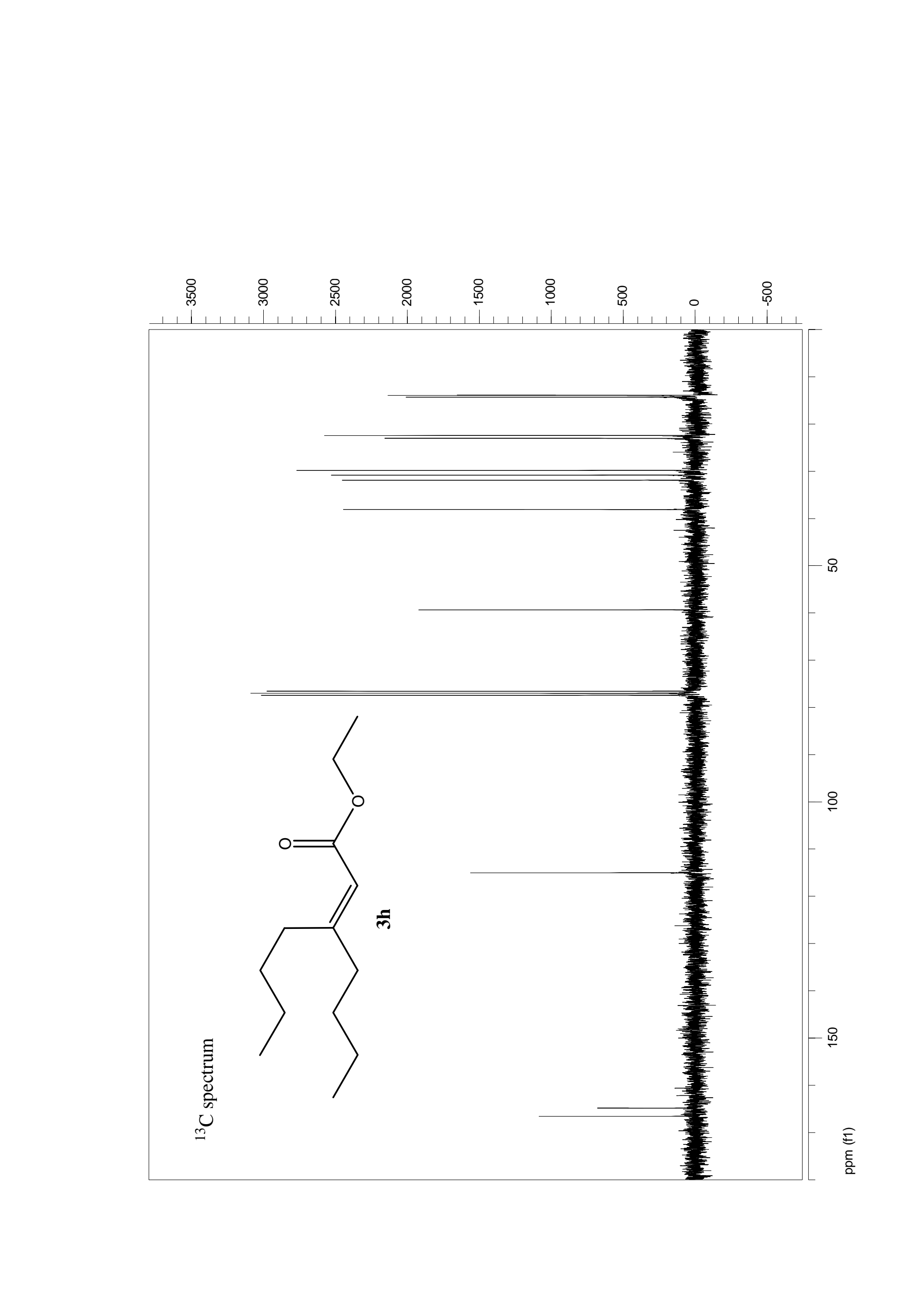




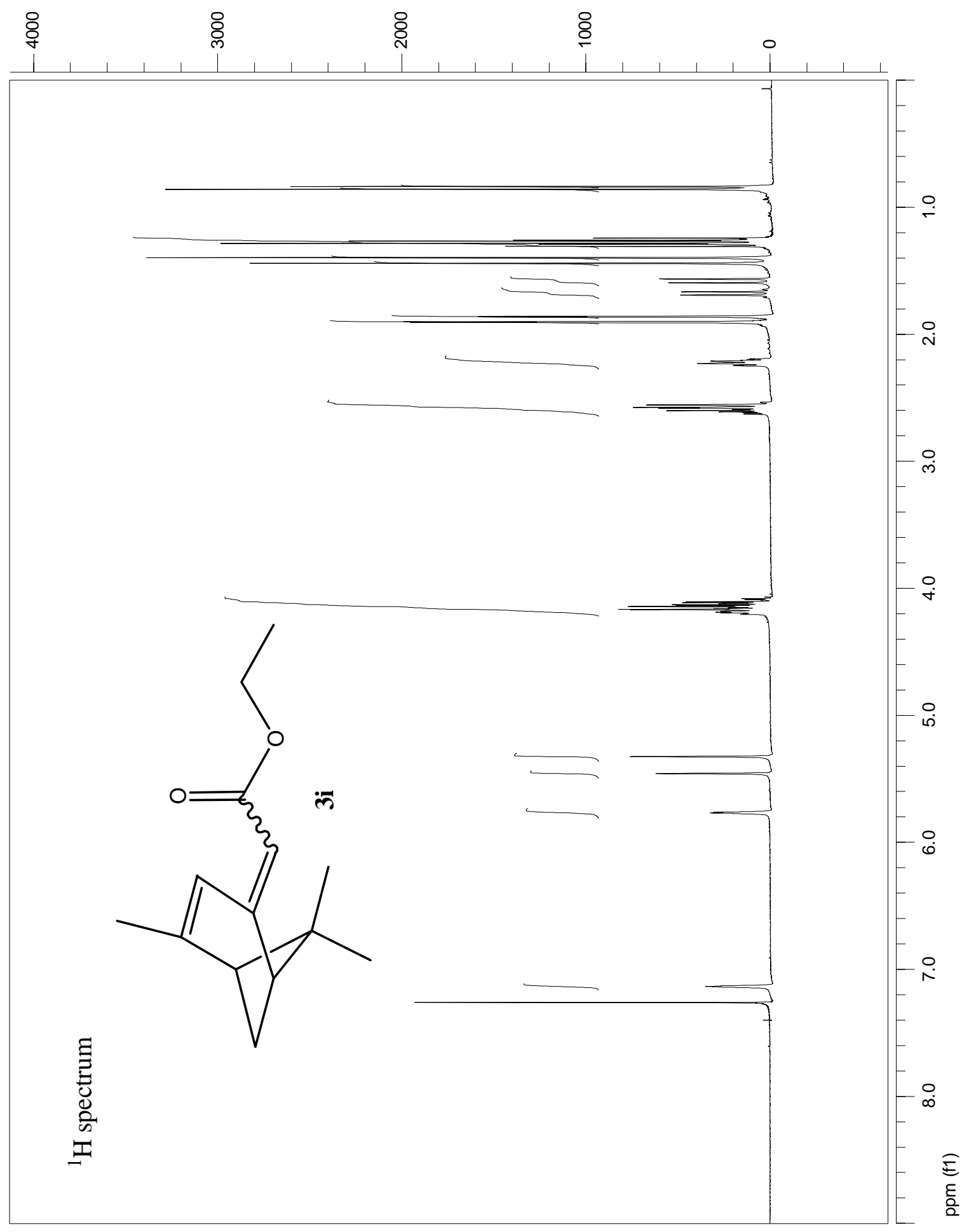




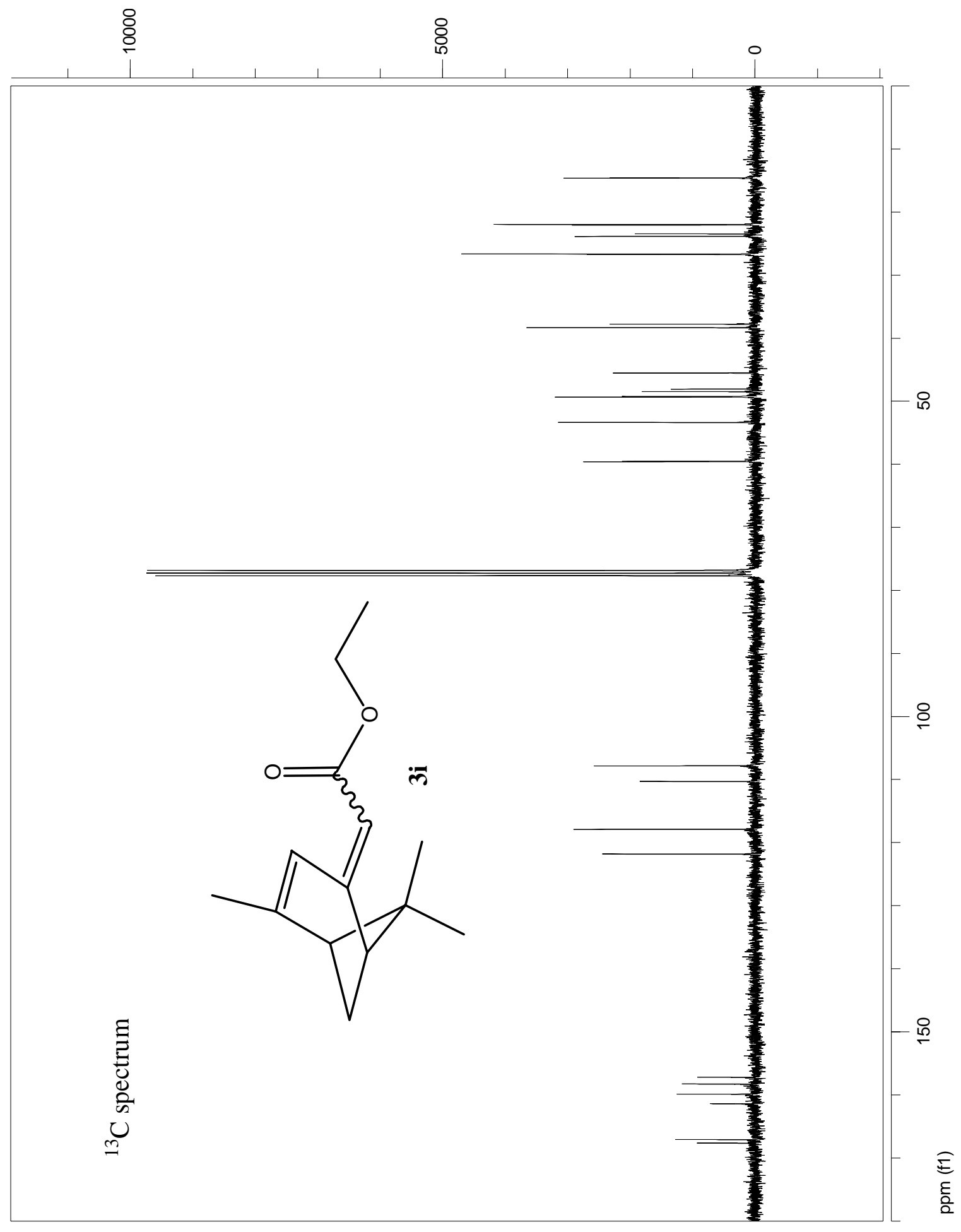

声 


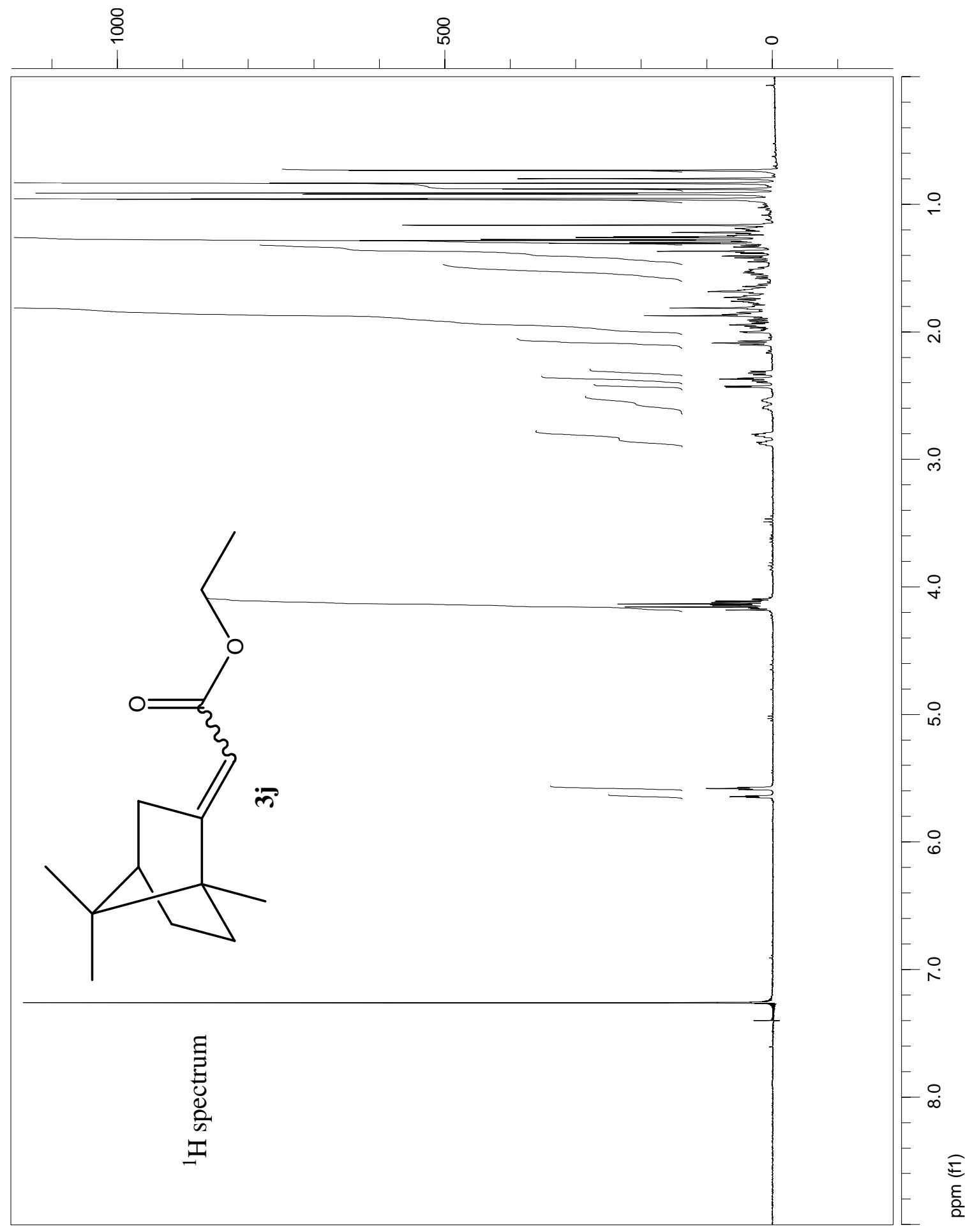




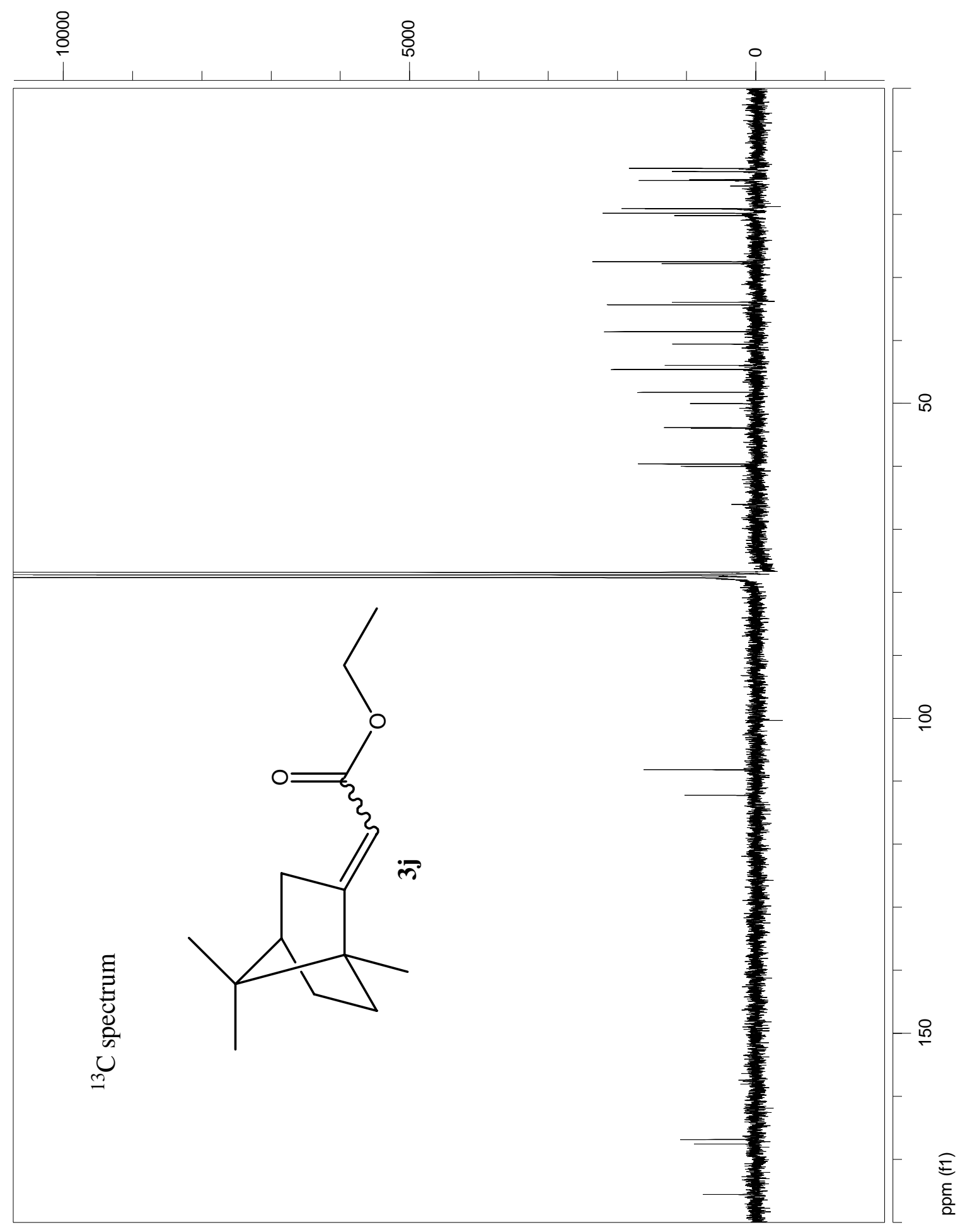

i 


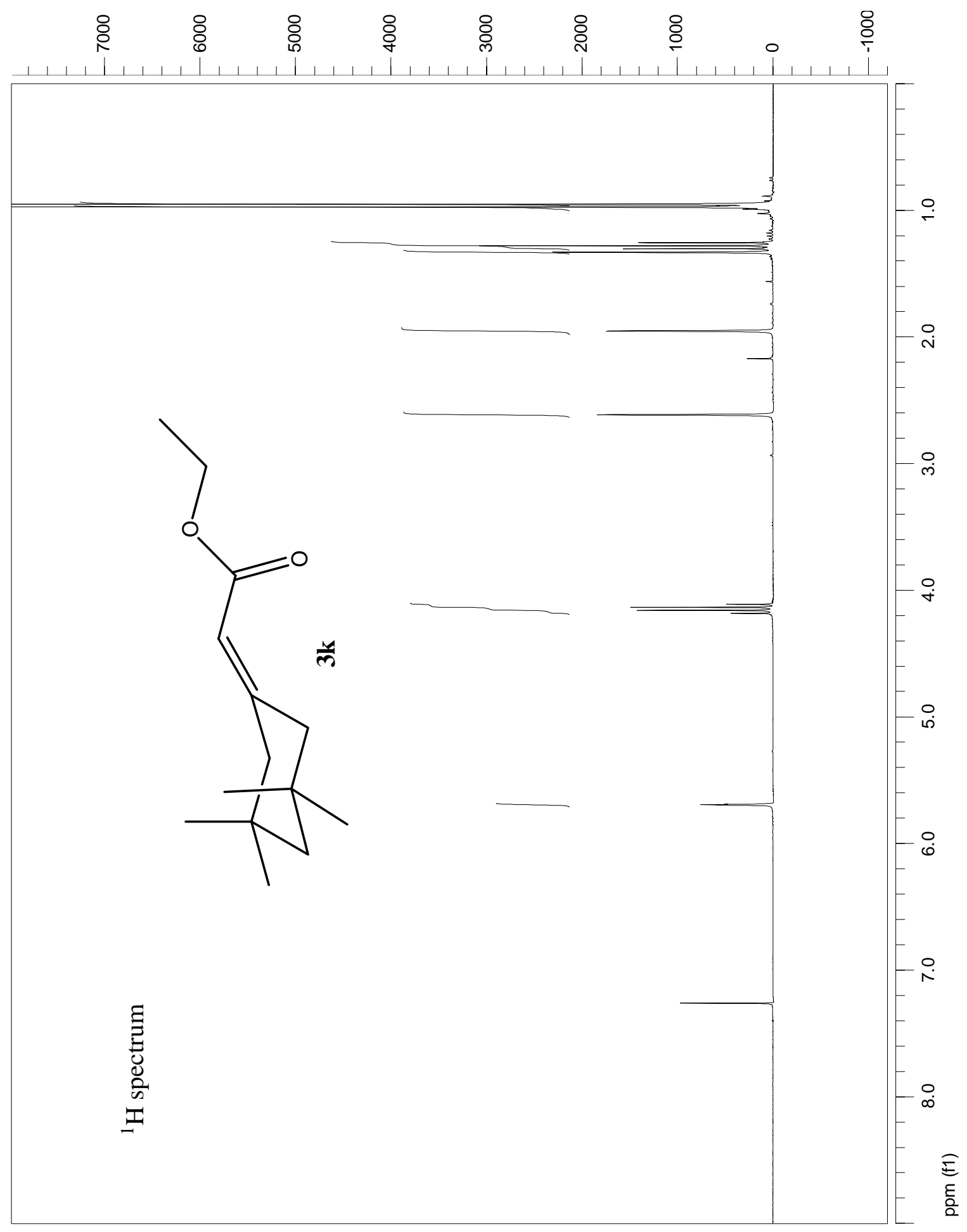

$\hat{n}$ 


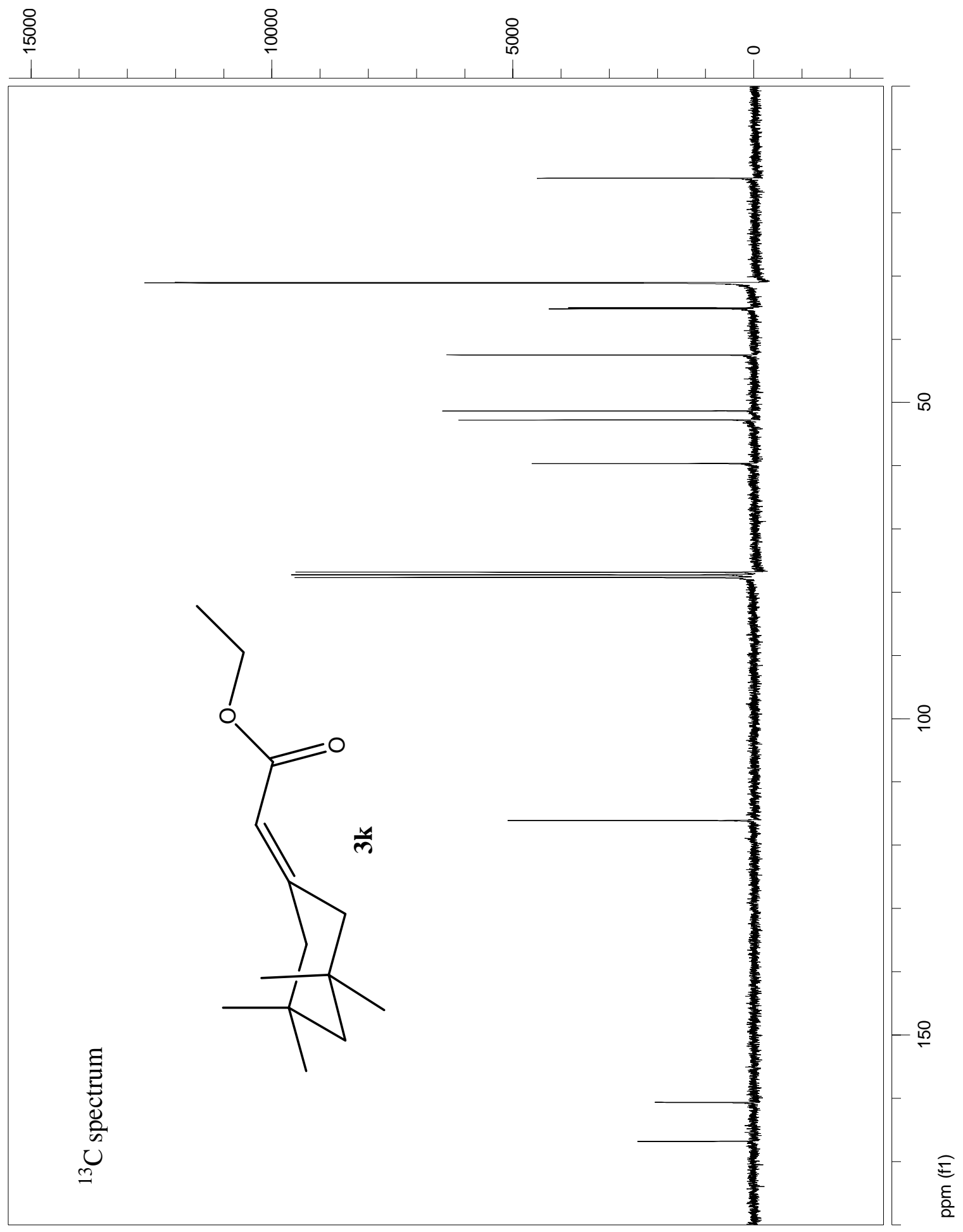

$\stackrel{\infty}{n}$ 